\title{
GROUP THEORETICAL APPROACHES TO VECTOR PARAME- TERIZATION OF ROTATIONS
}

\author{
ANDREAS MÜLLER
}

Communicated by Jan J. Slawianowski

\begin{abstract}
Known parametrizations of rotations are derived from the LIE group theoretical point of view considering the two groups $\mathrm{SO}(3)$ and $\mathrm{SU}(2)$. The concept of coordinates of the first and second kind for these groups is used to derive the axis and angle as well as the three-angle description of rotation matrices. With the homomorphism of the two groups the EULER parameter description arises from the axis and angle description of $\mathrm{SU}(2)$. Due to the topology of $\mathrm{SO}(3)$ any threeangle description gives only a local parametrization like EULER angles such that the mapping from their time derivatives to the algebra $\mathfrak{s o}(3)$, i.e., to the angular velocity tensor, exhibits singularities. All these parametrizations are based on the generation of the respective group by the exp map from their algebras. Alternatively the CAYLEY transformation also maps algebra elements to group elements. This fact is well know on $\mathrm{SO}(3)$ and yields a representation of rotation matrices in terms for RODRIGUES parameter, which is, however, not continuous. Generalizing this transformation to $\mathrm{SU}(2)$ allows for a singularity-free description of all rotations, which does not contain transcendental functions. While in the considered range the exponential map is of class $C^{\infty}$ the cay map on $\mathrm{SU}(2)$ is only of class $C^{1}$ and on $\mathrm{SO}(3)$ it is not even continuous. Simulation results exemplify the resultant numerical benefits for the simulation of rigid body dynamics. The problem caused by a lack of a continuous transformation from generalized accelerations to angular accelerations can be avoided for rigid body motions using the BOLZMANN-HAMEL equations.
\end{abstract}

\section{Contents}

1 Introduction $\quad 44$

2 The Group $\mathrm{SO}(3)$ and its Covering Group $\mathrm{SU}(2) \quad \mathbf{4 5}$

3 Representation of Rotations Using Canonical Coordinates 52

4 Vector Parametrization Based on CAYLEY transformations 57 
5 Angular Velocity and Acceleration

6 Computational Aspects

66

\section{Introduction}

The description of spatial rotations is a frequently addressed problem to which contributions were originally made by RODRIGUES, EULER, KLEIN and CAYLEY and many others. EULER and KLEIN independently derived equivalent four parametric descriptions of rotations. The complex valued CAYLEY-KLEIN parameter were independently introduced by CAYLEY [3-5] and KLEIN [13], [14] based on the work of RODRIGUES and EULER. CAYLEY used a projective description of the Riemannian sphere to the complex plane to derive a complex representation while EULER and RODRIGUES employed stereographic projections to gain real valued relations. It was already noticed by HAMILTON that all these descriptions are isomorphic to his quaternion algebra [9].

Rotations of three-dimensional vectors are naturally described by linear maps acting on representations of vectors in an orthonormal frame. In coordinate representation these maps are described by orthogonal matrices constituting a group, the special orthogonal group $\mathrm{SO}(3)$. Moreover, this group is a LIE group and its topology and representation is extensively investigated [1], [26-28], [30] . It is well known that $\mathrm{SO}(3)$ is connected but not simply connected. Also well studied is its covering group $\mathrm{SU}(2)$, which is simply connected. The LIE algebras of both groups are equivalent with respect to an isomorphism. Thus both groups are locally equivalent via a homomorphism.

Since the early work the set of possible parameterization contains the axis angle, three-angles, EULER and RODRIGUES parameter. But using a modified coordinate transformation to obtain the RODRIGUES parameter (RP) gives rise to a further parametrization, which is called the modified RP. It will be shown how this approach naturally follows from the CAYLEY transformation on $\mathrm{SU}(2)$ and fits into the LIE algebraic framework.

Vector-like descriptions of rotations based on the RP have attracted much attention in conjunction with efficient computer implementations [6-8], [29]. Numerical properties of conservative integration schemes using the CAYLEY transformation on $\mathrm{SO}(3)$ as well as its use for discretizations of finite rotations were investigated in [2] and [15]. The RP approach is especially useful for the description of small rotations as it is for the formulation of the kinematics of elastic bodies [12]. The 
LIE group property of spatial rotations and rigid body motions in general have been proven to be an important aspect that admits efficient symbolic computation [19-22]. Vector parameterization in particular are attractive since they give rise to purely algebraic and numerically advantageous descriptions of rotations.

In Section 2 the generation of $\mathrm{SO}(3)$ and $\mathrm{SU}(2)$ from their LIE algebras by the exponential map and the CAYLEY transformation is addressed. On $\mathrm{SO}(3)$ the CAYLEY transformation is well known but the CAYLEY transformation on SU(2) does not appear in the literature. The isomorphism of both algebras is then used in Section 3 to cast the adjoined actions of either groups on their algebra in the form of the conventional description of rotations, i.e., matrix times vector. In this way the four- and three-parametric descriptions based on the exponential map on $\mathrm{SO}(3)$ and $\mathrm{SU}(2)$ are obtained with canonical coordinates of the first or second kind, i.e., axis and angle or composition of relative rotations. Section 4 is concerned with parameterization that result from CAYLEY transformations and their relations to canonical coordinates on the respective group. On $\mathrm{SO}(3)$ these are known as RODRIGUES or CAYLEY parametrizations. But the CAYLEY transformation on $\mathrm{SU}(2)$ in conjunction with the group isomorphism of $\mathrm{SO}(3)$ and $\mathrm{SU}(2)$ yields a new parametrization, the modified RP. Vector fields in the respective algebras and on the parameter space are related in Section 5 to obtain angular velocities and accelerations in terms of the chosen parametrization. Section 6 concludes the paper with numerical issues and addresses the lack of a continuous mapping from accelerations of the modified RP to the angular acceleration vector. This drawback in the context of rigid body dynamics can, however, be overcome using the BOLZMANN-HAMEL equations instead of the LAGRANGIAN motion equations.

The LIE group theoretical background can be found in one of the excellent books like [25] or [10]. For further reading on differential geometric treatments of rotations the reader is referred to [1] and [23].

\section{The Group $\mathrm{SO}(3)$ and its Covering Group $\mathrm{SU}(2)$}

The set of all real valued orthogonal $3 \times 3$ matrices constitutes the special orthogonal group $\mathrm{SO}(3)$

$$
\mathrm{SO}(3)=\left\{A \in \operatorname{GL}(3, \mathbb{R}) ; A A^{T}=I_{3,3}, \operatorname{det} A=+1\right\} .
$$

It is a three-dimensional LIE group with LIE algebra $\mathfrak{s o}(3)$, the set of all real valued $3 \times 3$ skew symmetric matrices

$$
\mathfrak{s o}(3)=\left\{\omega \in \mathbb{R}^{3,3} ; \omega+\omega^{T}=0\right\} .
$$


As such $\mathrm{SO}(3)$ is generated by the LIE algebra $\mathfrak{s o}(3)$ via the exponential map $\exp : \mathfrak{s o}(3) \rightarrow \mathrm{SO}(3)$. A basis for $\mathfrak{s o}(3)$ such that $\mathfrak{s o}(3)=\operatorname{span}\left(L_{1}, L_{2}, L_{3}\right)_{\mathbb{R}}$ is given by $L_{i}=\left(-\varepsilon_{i j k}\right) \in \mathbb{R}^{3,3}, i=1,2,3$ or in matrix form

$$
L_{1}=\left(\begin{array}{rrr}
0 & 0 & 0 \\
0 & 0 & -1 \\
0 & 1 & 0
\end{array}\right), \quad L_{2}=\left(\begin{array}{rrr}
0 & 0 & 1 \\
0 & 0 & 0 \\
-1 & 0 & 0
\end{array}\right), \quad L_{3}=\left(\begin{array}{rrr}
0 & -1 & 0 \\
1 & 0 & 0 \\
0 & 0 & 0
\end{array}\right) \text {. }
$$

If the generating LIE algebra element for $R \in \mathrm{SO}(3)$ has the form ${ }^{1}$

$$
\begin{aligned}
\omega_{X} & =X^{i} L_{i}, \quad \omega_{X} \in \mathfrak{s o}(3), \quad X^{i} \in \mathbb{R} \\
\left(\omega_{X}\right)_{i j} & =-\varepsilon_{i j k} X^{k}
\end{aligned}
$$

such that $R=\exp \left(X^{i} L_{i}\right)$ then the components $X^{i}, i=1,2,3$ are canonical coordinates of the first kind $(\mathrm{C} 1 \mathrm{~K})$. The group $\mathrm{SO}(3)$ is a connected threedimensional LIE group and can hence be locally expressed as product of three one-dimensional subgroups such that $R=\exp \left(\theta_{1} L_{i}\right) \exp \left(\theta_{2} L_{j}\right) \exp \left(\theta_{3} L_{k}\right)$, $i \neq j \neq k$ where therein $\theta_{i}, i=1,2,3$ are canonical coordinates of the second kind $(\mathrm{C} 2 \mathrm{~K})$ on the LIE group $\mathrm{SO}(3)$ [17]. The concept of coordinates of the first and second kind is of special importance for the description of spatial rotations. Although $\mathrm{SO}(3)$ is connected, it is not simply connected and thus any product representation cannot be global, i.e., for any such representation there exists a point where the exponential map parameterized by $\mathrm{C} 2 \mathrm{~K}$ is not bijective. Examples for $\mathrm{C} 2 \mathrm{~K}$ on $\mathrm{SO}(3)$ are every three angles used to describe spatial rotations, e.g. EULER or BRYANT angles. C1K are the rotation axis and angle. On $\mathfrak{s o}(3)$ as matrix LIE algebra the LIE bracket is the matrix commutator and it holds that

$$
\left[L_{i}, L_{j}\right]=L_{i} L_{j}-L_{j} L_{i}=\varepsilon_{i j k} L_{k}
$$

thus the structure constants of $\mathfrak{s o}(3)$ are given by the LEVI-CIVITA symbol. The LIE bracket defines a linear map $\operatorname{ad}_{\omega}: \mathfrak{s o}(3) \rightarrow \mathfrak{s o}(3)$ for fixed $\omega, \operatorname{ad}_{\omega}(\eta):=$ $[\omega, \eta]$. A norm on $\mathfrak{s o}(3)$ is given by

$$
\left\|\omega_{X}\right\|_{\mathfrak{s o}(3)}^{2}=\operatorname{tr}\left(\omega_{X}^{T} \omega_{X}\right)=\|X\|_{\mathbb{R}^{3}}^{2}
$$

where $X:=\left(X^{1}, X^{2}, X^{3}\right),\|X\|_{\mathbb{R}^{3}}^{2}=\langle X, X\rangle_{\mathbb{R}^{3}}=X^{T} X$ is the standard inner product on $\mathbb{R}^{3}$. For simplicity the subscripts in (6) will be dropped if there is no danger of confusion. For $\mathfrak{s o}(3)$ the exponential map is explicitly expressed

\footnotetext{
${ }^{1}$ Convention: summation over repeated indices on different levels.
} 
using the coordinates of the first kind $X^{i}$ by the well known EULER- RODRIGUES formula

$$
R(X):=\exp \omega_{X}=I+\frac{\sin \left\|\omega_{X}\right\|}{\left\|\omega_{X}\right\|} \omega_{X}+\frac{1-\cos \left\|\omega_{X}\right\|}{\left\|\omega_{X}\right\|^{2}} \omega_{X}^{2}, \quad \omega_{X} \in \mathfrak{s o}(3) .
$$

If $\left\|\omega_{\bar{X}}\right\|=1$ then (7) yields a rotation matrix describing a rotation about the axis $\bar{X} \in \mathbb{R}^{3}$ and angle $\theta \in(-\pi, \pi]^{2}$

$$
R(\theta, \bar{X}):=\exp \left(\theta \omega_{\bar{X}}\right)=I+\sin \theta \omega_{\bar{X}}+(1-\cos \theta) \omega_{\bar{X}}^{2} .
$$

The $\mathrm{SO}(3)$ group acts on its LIE algebra via the adjoint map $\mathrm{Ad}: \mathrm{SO}(3) \times \mathfrak{s o}(3) \rightarrow$ $\mathfrak{s o}(3)$ which describes how group elements act on algebra elements. A basic result from the theory of LIE groups is the relation (cf. [17]) $\exp \operatorname{ad}_{\omega} \eta=\operatorname{Ad}_{\exp \omega} \eta$ with $\omega, \eta \in \mathfrak{s o}(3)$.

Proposition 1. If $R=\exp (\omega)$ and $\omega, \eta \in \mathfrak{s o}(3)$, the adjoint action $R$ on $\eta$ is explicitly given by

$$
\operatorname{Ad}_{R} \eta=\exp \operatorname{ad}_{\omega} \eta=\left(I+\frac{\sin \|\omega\|}{\|\omega\|} \operatorname{ad}_{\omega}+\frac{1-\cos \|\omega\|}{\|\omega\|^{2}} \operatorname{ad}_{\omega}^{2}\right) \eta
$$

where $\operatorname{ad}_{\omega}^{i}$ is the $i$-fold composition of the operator $\operatorname{ad}_{\omega}$.

Proof: Iterative application of $\operatorname{ad}_{\omega}$ together with $\omega^{3}=-\|\omega\|^{2} \omega$ yields

$$
\operatorname{ad}_{\omega}^{j} \eta= \begin{cases}(-1)^{k}\|\omega\|^{2 k} \operatorname{ad}_{\omega} \eta, & j=2 k+1 \\ (-1)^{k}\|\omega\|^{2 k} \operatorname{ad}_{\omega}^{2} \eta, & j=2 k+2, \quad k=0,1,2, \ldots\end{cases}
$$

and substitution into the series expansion of exp gives the result

$$
\begin{aligned}
\exp [\omega, \eta] & =v+\sum_{k=0}^{\infty}(-1)^{k}\|\omega\|^{2 k}\left(\frac{\operatorname{ad}_{\omega} \eta}{(2 k+1) !}+\frac{\operatorname{ad}_{\omega}^{2} \eta}{(2 k+2) !}\right) \\
& =\left(I+\frac{\sin \|\omega\|}{\|\omega\|} \operatorname{ad}_{\omega}+\frac{(1-\cos \|\omega\|)}{\|\omega\|^{2}} \operatorname{ad}_{\omega}^{2}\right) \eta .
\end{aligned}
$$

By the bilinearity of ad it follows that for $\left\|\omega_{\bar{X}}\right\|=1, \operatorname{Ad}_{\exp \theta \omega_{\bar{X}}} \eta=\eta+$ $\sin \theta\left[\omega_{\bar{X}}, \eta\right]+(1-\cos \theta)\left[\omega_{\bar{X}},\left[\omega_{\bar{X}}, \eta\right]\right]$.

Another way to map algebra elements to group elements which in contrast to relations (9) does not contain transcendental functions is the CAYLEY transformation.

\footnotetext{
${ }^{2}$ Let $a \in \mathbb{R}^{3}$ be an arbitrary vector then $\bar{a}$ denotes the normalized vector: $\bar{a}:=a /\|a\|$
} 
Lemma 2. The CAYLEY transformation cay: $\omega \longmapsto(I-\omega)^{-1}(I+\omega)$, for $\omega \in \mathfrak{s o}(3)$ is a map from $\mathfrak{s o}(3)$ to $\mathrm{SO}(3)$ and can be expressed as

$$
\operatorname{cay}(\omega)=(I-\omega)^{-1}(I+\omega)=I+\frac{2}{1+\|\omega\|^{2}}\left(\omega+\omega^{2}\right), \quad \omega \in \mathfrak{s o}(3) \text {. }
$$

Proof: Using the Neumann series $(I-\omega)^{-1}=I+\omega+\omega^{2}+\omega^{3}+\cdots$ with the relation $\omega^{3}=-\|\omega\|^{2} \omega$ for higher order terms (11) can be written as

$$
\begin{aligned}
\operatorname{cay}(\omega) & =\left(I+\omega+\omega^{2}+\omega^{3}+\cdots\right)(I+\omega) \\
& =I+2\left(1-\|\omega\|^{2}+\|\omega\|^{4}-\|\omega\|^{6}+\cdots\right)\left(\omega+\omega^{2}\right) \\
& =I+\frac{2}{1+\|\omega\|^{2}}\left(\omega+\omega^{2}\right) .
\end{aligned}
$$

$R(\omega) \in \mathrm{SO}(3)$ if and only if $R(\omega)^{T} R(\omega)=I$ and $\operatorname{det} R(\omega)=1$. With (13) it holds that

$$
R^{T} R=I+4\left(1+\|\omega\|^{2}\right)^{-1} \omega^{2}+4\left(1+\|\omega\|^{2}\right)^{-2}\left(\omega^{4}-\omega^{2}\right)=I
$$

and $\operatorname{det}(I+\omega)=\Pi_{i=1}^{3} \lambda_{i}(I+\omega)$ and $\operatorname{det}(I-\omega)^{-1}=1 / \operatorname{det}(I-\omega)$, where $\lambda_{i}(A)$ is the $i$-th eigenvalue of $A$. From the power expansion $I+\omega=\omega^{0}+\omega$ and $I-\omega=(-\omega)^{0}-\omega$ follows that $\lambda_{i}(I+\omega)=1+\lambda_{i}(\omega)$ and $\lambda_{i}(I-\omega)=$ $1+\lambda_{i}(-\omega)$ respectively. The characteristic equation $\omega^{3}+\|\omega\|^{2} \omega=0$ yields the eigenvalues $\lambda_{1}(\omega)=0, \lambda_{2,3}(\omega)= \pm \mathrm{i}\|\omega\|$ and $\lambda_{1}(I+\omega)=1, \lambda_{2,3}(I+\omega)=$ $1 \pm \mathrm{i}\|\omega\|$. Thus $\operatorname{det}(I+\omega)=1+\|\omega\|^{2}$. Proceeding in the same way it follows that $\operatorname{det}(I-\omega)=1+\|\omega\|^{2}$ and finally $\operatorname{det} R(\omega)=1$.

Let $\omega_{c}=c^{i} L_{i}$ and $R(c):=$ cay $\left(\omega_{c}\right)$ with $c \in \mathbb{R}^{3}$ then the vector $c$ is known as the RODRIGUES vector of $R$ whose components $c^{i}, i=1,2,3$ will be referred to as RODRIGUES parameter (RP) throughout. If again $\left\|\omega_{\bar{X}}\right\|=1$ then $R(\gamma, \bar{X}):=$ cay $\left(\gamma \omega_{\bar{X}}\right)=I+\frac{2}{1+\gamma_{\bar{X}}^{2}}\left(\gamma \omega_{\bar{X}}+\gamma^{2} \omega_{\bar{X}}^{2}\right)$ is an orthogonal matrix determined by the parameter $\gamma$ and axis $\bar{X}$ via (4). Therein the real parameter $\gamma$ will be called the CAYLEY parameter (CP) for a rotation about $\bar{X}$. It should be noticed that some authors refer to the components $c^{i}$ of the RODRIGUES vector as CAYLEY parameter [17] while others call them RODRIGUES parameter [18]. As will be seen later, the RoDRIGUES vector $c \in \mathbb{R}^{3}$ and the vector $X \in \mathbb{R}^{3}$ in (7) are related by a coordinate transformation in $\mathbb{R}^{3}$ as it should be expected since the exp map is locally surjective for a compact group. 
The (simply connected) covering group of $\mathrm{SO}(3)$ is the special unitary group $\mathrm{SU}(2)$

$$
\mathrm{SU}(2)=\left\{U \in \mathrm{GL}(\mathbb{C}, 2) ; U U^{*}=I_{2,2}\right\} .
$$

The unitarity condition on $U \in \mathrm{SU}(2)$ demands the special form

$$
U=\left(\begin{array}{rr}
a & b \\
-\bar{b} & \bar{a}
\end{array}\right)
$$

with complex values $a, b \in \mathbb{C}$ such that $|a|^{2}+|b|^{2}=1$. These numbers $a, b$ are called CAYLEY-KLEIN parameters [1]. The generating LIE algebra of SU(2) is $\mathfrak{s u}(2)$, the set of all skew Hermitian $2 \times 2$ matrices

$$
\mathfrak{s u}(2)=\left\{u \in \mathbb{C}^{2,2} ; u+u^{*}=0\right\} .
$$

Since for $u \in \mathfrak{s u}(2), u=-u^{*}$ it follows that $\operatorname{tr}(u)=0$. A basis for $\mathfrak{s u}(2)$ such that $\mathfrak{s u}(2)=\operatorname{span}_{\mathbb{R}}\left(E_{1}, E_{2}, E_{3}\right)$ is given by

$$
E_{k}=\frac{\mathrm{i}}{2} \sigma_{k}, \quad k=1,2,3
$$

where

$$
\sigma_{1}=\left(\begin{array}{ll}
0 & 1 \\
1 & 0
\end{array}\right), \quad \sigma_{2}=\left(\begin{array}{rr}
0 & -\mathrm{i} \\
\mathrm{i} & 0
\end{array}\right), \quad \sigma_{3}=\left(\begin{array}{rr}
1 & 0 \\
0 & -1
\end{array}\right)
$$

are the PAULI matrices. In analogy to (4) every element $u \in \mathfrak{s u}(2)$ can be expressed as a linear combination of $E_{i}, i=1,2,3$ with real coefficients $X^{i}$

$$
u_{X}=X^{i} E_{i}, u_{X} \in \mathfrak{s u}(2), \quad X^{i} \in \mathbb{R}, \quad i=1,2,3 .
$$

The LIE bracket for the matrix algebra $\mathfrak{s u}(2)$ is the matrix commutator

$$
[u, v]=u v-v u, \quad u, v \in \mathfrak{s u}(2) .
$$

For $\mathfrak{s u}(2)$ the structure constants are due to the relations $E_{1} E_{2}=\frac{1}{2} E_{3}, E_{3} E_{1}=$ $\frac{1}{2} E_{2}, E_{2} E_{3}=\frac{1}{2} E_{1}$ and thus

$$
\left[E_{i}, E_{j}\right]=\varepsilon_{i j k} E_{k}
$$

are again determined by the LEVI-CIVITA symbol. A norm on $\mathfrak{s u}(2)$ can be introduced as

$$
\|u\|^{2}:=\operatorname{det} u
$$


such that with $u_{X}=X^{i} E_{i}$ it holds $\left\|u_{X}\right\|_{\mathfrak{s u}(2)}^{2}=\|X\|_{\mathbb{R}^{3}}^{2}$. A remarkable property of $\mathrm{SU}(2)$ is that any $U \in \mathrm{SU}(2)$ can be expressed as a linear combination in the basis $\left\{P_{0}, P_{1}, P_{2}, P_{3}\right\}$, i.e.,

$$
U=p^{\nu} P_{\nu}
$$

with the condition $\sum_{\nu=0}^{3}\left(p^{\nu}\right)^{2}=1$. The basis elements are given in terms of the basis on its algebra $\mathfrak{s u}(2)$

$$
P_{\nu}= \begin{cases}I_{\mathrm{SU}(2)} & \nu=0 \\ 2 \delta_{\nu i} E_{i} & \nu=1,2,3 .\end{cases}
$$

The real parameter $p^{\nu} \in \mathbb{R}, \nu=0,1,2,3$ in (21) are the EULER parameter (EP) associated with a rotation about the axis $\bar{X} \in \mathbb{R}^{3},\|\bar{X}\|=1$. They are related to the CAYLEY-KLEIN parameter by

$$
a=p^{0}+\mathrm{i} p^{3}, \quad b=p^{2}+\mathrm{i} p^{1} .
$$

The fact that $\mathrm{SU}(2)$ elements can be expanded as a linear combination (21) finds it underlying reason in the existence of an homomorphism of $\mathrm{SU}(2)$ and the quaternion algebra. As a result the multiplication law for EP is determined by that of quaternions and hence the group multiplication in $\mathrm{SU}(2)$ is governed by the quaternion multiplication.

Lemma 3. For any $u \in \mathfrak{s u}(2)$ it holds

$$
\exp u=I \cos \left\|\frac{u}{2}\right\|+2 \sin \left\|\frac{u}{2}\right\| \frac{u}{\|u\|} .
$$

Proof: The characteristic equation for $u=X^{i} E_{i} \in \mathfrak{s u}(2)$ is $\|u\|^{2}+4 \lambda^{2}=0$ and hence with the CAYLEY- HAMiLTON theorem it also holds $u^{2}=-\frac{1}{4}\|u\|^{2} I$. It can be shown that $u^{j}=(-1)^{k} \frac{1}{2^{2 k}}\|u\|^{2 k} u, j=2 k+1$ and $u^{j}=(-1)^{k} \frac{1}{2^{2 k}}\|u\|^{2 k} I$, $j=2 k, k=0,1,2, \ldots$ which yields the desired result

$$
\begin{aligned}
\exp u & =\sum_{k=0}^{\infty}(-1)^{k}\left(\frac{\|u\|^{2 k}}{2^{2 k}(2 k) !} I+\frac{\|u\|^{2 k+1}}{2^{2 k+1}(2 k+1) !} u\right) \\
& =I \cos \left\|\frac{u}{2}\right\|+\frac{2}{\|u\|} \sin \left\|\frac{u}{2}\right\| u
\end{aligned}
$$

and taking into account that $\left\|u_{\bar{X}}\right\|=1$ the representation of $U \in \mathrm{SU}(2)$ in terms of coordinates of the first kind is

$$
U(\theta, \bar{X}):=\exp \theta u_{\bar{X}}=I \cos \frac{\theta}{2}+2 \sin \frac{\theta}{2} u_{\bar{X}} .
$$


The general result $\exp \operatorname{ad}_{u} v=\operatorname{Ad}_{\exp u} v, u, v \in \mathfrak{s u}(2)$ with the bilinear map $\operatorname{ad}_{u} v=[u, v]$ gives rise to an explicit formulation for $\mathfrak{s u}(2)$ such as (9).

Lemma 4. For any $u, v \in \mathfrak{s u}(2)$ the adjoint map $\operatorname{Ad}_{\exp u}: \mathfrak{s u}(2) \rightarrow \mathfrak{s u}(2)$ can be expressed as

$$
\exp \operatorname{ad}_{u} v=v+\frac{\sin \|u\|}{\|u\|} \operatorname{ad}_{u} v+\frac{1-\cos \|u\|}{\|u\|^{2}} \operatorname{ad}_{u}^{2} v .
$$

Proof: Using the relation for higher order terms $u^{i}$ above gives

$$
\operatorname{ad}_{u}^{j} v=\left\{\begin{array}{l}
(-1)^{k}\|u\|^{2 k} \operatorname{ad}_{u} v, \quad j=2 k+1 \\
(-1)^{k}\|u\|^{2 k} \operatorname{ad}_{u}^{2} v, \quad j=2 k+2, \quad k=0,1,2, \ldots
\end{array}\right.
$$

and the series expansion of exp yields

$$
\begin{aligned}
\exp \operatorname{ad}_{u} v & =v+\sum_{k=0}^{\infty}(-1)^{k}\|\omega\|^{2 k}\left(\frac{\operatorname{ad}_{u} v}{(2 k+1) !}+\frac{\operatorname{ad}_{u}^{2} v}{(2 k+2) !}\right) \\
& =\left(I+\frac{\sin \|u\|}{\|u\|} \operatorname{ad}_{u}+\frac{1-\cos \|u\|}{\|u\|^{2}} \operatorname{ad}_{u}^{2}\right) v
\end{aligned}
$$

Presumed $\left\|u_{\bar{X}}\right\|=1$ it follows with the bilinearity of $\operatorname{ad}_{u} v$ that

$$
\operatorname{Ad}_{\exp \theta u_{\bar{X}}} v=v+\sin \theta\left[u_{\bar{X}}, v\right]+(1-\cos \theta)\left[u_{\bar{X}},\left[u_{\bar{X}}, v\right]\right] .
$$

As for $\mathrm{SO}(3)$ the CAYLEY transformation maps elements of the LIE algebra $\mathfrak{s u}(2)$ to elements in the group.

Theorem 5. The transformation cay: $u \longmapsto(I-u)^{-1}(I+u), u \in \mathfrak{s u}(2) d e$ fines a map from $\mathfrak{s u}(2)$ to $\mathrm{SU}(2)$ and has the explicit form

$$
\operatorname{cay}(u)=(I-u)^{-1}(I+u)=\frac{1-\left\|\frac{u}{2}\right\|^{2}}{1+\left\|\frac{u}{2}\right\|^{2}} I+\frac{2}{1+\left\|\frac{u}{2}\right\|^{2}} u, \quad u \in \mathfrak{s u}(2) \text {. }
$$

Proof: The series expansion $(I-u)^{-1}=I+u+u^{2}+u^{3}+\cdots$ yields $(I-u)^{-1}=$ $\frac{4}{4+\|u\|^{2}}(I+u)$ and thus

$$
\begin{aligned}
U & =\operatorname{cay}(u)=(I-u)^{-1}(I+u) \\
& =\frac{4}{4+\|u\|^{2}}(I+u)^{2}=\frac{1-\left\|\frac{u}{2}\right\|^{2}}{1+\left\|\frac{u}{2}\right\|^{2}} I+\frac{2}{1+\left\|\frac{u}{2}\right\|^{2}} u .
\end{aligned}
$$


$U \in \mathrm{SU}(2)$ if and only if $U^{*} U=I$ and $\operatorname{det} U=1$. With $u^{*}=-u$ it follows that

$$
\begin{aligned}
U^{*} U & =16\left(4+\|u\|^{2}\right)^{-2}(I-u)^{2}(I+u)^{2} \\
& =\left(16+8\|u\|^{2}+\|u\|^{4}\right)\left(4+\|u\|^{2}\right)^{-2} I=I .
\end{aligned}
$$

From the characteristic equation $\|u\|^{2}+4 \lambda^{2}=0$ the eigenvalues of $u$ can be deduced as $\lambda_{1,2}= \pm \frac{\mathrm{i}}{2}\|u\|$ and $\lambda_{i}(I+u)=1 \pm \frac{\mathrm{i}}{2}\|u\|$. Hence it follows that $\operatorname{det}\left((I-u)^{-1}(I+u)\right)=\frac{4}{4+\|u\|^{2}}\left(1+\frac{1}{4}\|u\|^{2}\right)=1$.

Alternatively $U=$ cay $(u)$ can be expressed as a product

$$
U(u)=U^{\frac{1}{2}}(u) \cdot U^{\frac{1}{2}}(u) \text { with } U^{\frac{1}{2}}(u)=\left(1+\frac{1}{2}\|u\|^{2}\right)^{-\frac{1}{2}}(I+u) .
$$

Let $u_{a} \in \mathrm{SU}(2)$ be expressed as $u_{a}=2 a^{i} E_{i}$, with $a \in \mathbb{R}^{3}$ such that $U(a):=$ cay $\left(u_{a}\right)$. Further set $u=\bar{X}^{i} E_{i}$ with $\left\|u_{\bar{X}}\right\|_{\mathfrak{s u}(2)}=\|X\|_{\mathbb{R}^{3}}=1$ and introduce a real parameter $\alpha$ such that

$$
U(\alpha, \bar{X}):=\operatorname{cay}\left(2 \alpha u_{\bar{X}}\right)=\frac{1-\alpha^{2}}{1+\alpha^{2}} I+\frac{4 \alpha}{1+\alpha^{2}} u .
$$

Definition 6. Let $u_{a}=a^{i} E_{i}$ and $U(a)=$ cay $\left(2 u_{a}\right)$. The vector $a=\left(a^{i}\right) \in \mathbb{R}^{3}$ is called the modified RODRIGUES vector of $U$. If $U(\alpha, \bar{X})=$ cay $\left(2 \alpha u_{\bar{X}}\right)$ with $\left\|u_{\bar{X}}\right\|=1$, then $\alpha \in \mathbb{R}$ is called the modified CAYLEY parameter of $U$.

The above introduced parameters $\alpha$ and $a$ are arbitrary and so their respective definition was chosen in order to simplify the later expressions for rotation matrices avoiding the factor 2. Modified CP and RP are not canonical coordinates by them self but they result from those by a coordinate transformation on $\mathbb{R}^{3}$.

\section{Representation of Rotations Using Canonical Coordinates}

In the context of rotations the algebras of interest are $\mathbb{R}^{3}, \mathfrak{s o}(3)$ and $\mathfrak{s u}(2)$. Relations of these algebras and of the two groups $\mathrm{SU}(2)$ and $\mathrm{SO}(3)$ give rise to different parametrization of rotation expressed in terms of $\mathrm{C} 1 \mathrm{~K}$ or $\mathrm{C} 2 \mathrm{~K}$ on either group. By a coordinate transformation on the respective group, $\mathrm{SO}(3)$ and $\mathrm{SU}(2)$ can be parameterized in terms of the respective CPs as shown in the next section. The vector space $\mathbb{R}^{3}$ is a LIE algebra where the cross product represents the LIE bracket $[X, Y]_{\mathbb{R}^{3}}:=X \times Y, X, Y \in \mathbb{R}^{3}$, where in an orthonormal basis it holds 
for $Z=X \times Y$ that $Z^{i}=\frac{1}{2} \varepsilon^{i}{ }_{j k} X^{j} Y^{k}$ and thus the structure constants of this LIE algebra are given by the LEVI-CIVITA symbol. The LIE algebras $\mathfrak{s o}(3)$ and $\mathbb{R}^{3}$ are isomorphic via the isomorphism

$$
\pi_{1}: \mathfrak{s o}(3) \rightarrow \mathbb{R}^{3}, \quad \omega_{X}=X^{i} L_{i} \mapsto X
$$

with $\omega_{X}=\left(-\varepsilon_{i j k} X^{k}\right)$ and $X^{k}=-\frac{1}{2} \varepsilon^{k i j} \omega_{i j}$. I.e. $\pi_{1} \circ\left[\omega_{X}, \omega_{Y}\right]_{\mathfrak{s o}(3)}=$ $\left[\pi_{1}\left(\omega_{X}\right), \pi_{1}\left(\omega_{Y}\right)\right]_{\mathbb{R}^{3}}=[X, Y]_{\mathbb{R}^{3}}$, with $\omega_{X}=X^{i} L_{i}$ and $\omega_{Y}=Y^{i} L_{i}$. Analogously $\mathfrak{s u}(2)$ is isomorphic to $\mathbb{R}^{3}$ via

$$
\pi_{2}: \mathfrak{s u}(2) \rightarrow \mathbb{R}^{3}, \quad u_{X}=X^{i} E_{i} \mapsto X
$$

with $X^{k}=-2 \operatorname{tr}\left(u_{X} E_{k}\right)$, such that $\pi_{2} \circ\left[u_{X}, u_{Y}\right]_{\mathfrak{s u}(2)}=\left[\pi_{2}\left(u_{X}\right), \pi_{2}\left(u_{Y}\right)\right]_{\mathbb{R}^{3}}=$ $[X, Y]_{\mathbb{R}^{3}}$. The last equation follows from the relations $E_{i} E_{j}=\varepsilon_{i j k} E_{k}-\frac{1}{4} \delta_{i j}$ which produce $\operatorname{tr}\left(E_{i} E_{j}\right)=-\frac{1}{2} \delta_{i j}$. Hence $\pi_{3}:=\pi_{1}^{-1} \circ \pi_{2}$ defines an isomorphism of $\mathfrak{s o}(3)$ and $\mathfrak{s u}(2)$, such that

$$
\pi_{3} \circ\left[u_{X}, u_{Y}\right]_{\mathfrak{s u}(2)}=\left[\pi_{3}\left(u_{X}\right), \pi_{3}\left(u_{Y}\right)\right]_{\mathfrak{s o}(3)}=\left[\omega_{X}, \omega_{Y}\right]_{\mathfrak{s o}(3)} .
$$

The product representation of the cross product on $\mathbb{R}^{3}$ is obtained from the LIEproduct on $\mathfrak{s o}(3)$ using $\pi_{1}$

$$
\begin{aligned}
Z= & \pi_{1} \circ\left[\omega_{X}, \omega_{Y}\right]_{\mathfrak{s o}(3)}=\pi_{1}\left(\omega_{X}\right) \times \pi_{1}\left(\omega_{Y}\right)=X \times Y=\omega_{X} Y \\
& X, Y, Z \in \mathbb{R}^{3}, \omega_{X}, \omega_{Y} \in \mathfrak{s o}(3)
\end{aligned}
$$

which can be deduced along $Y^{k}=-\frac{1}{2} \varepsilon^{k i j}\left(\omega_{Y}\right)_{i j}$ from

$$
\begin{aligned}
Z^{i} & =\frac{1}{8} \varepsilon^{i}{ }_{j k} \varepsilon^{j m n} \varepsilon^{k r s}\left(\omega_{X}\right)_{m n}\left(\omega_{Y}\right)_{r s} \\
& =\frac{1}{4}\left(\delta_{k}^{m} \delta^{i n}-\delta_{k}^{n} \delta^{i m}\right) \varepsilon^{k r s}\left(\omega_{X}\right)_{m n}\left(\omega_{Y}\right)_{r s} \\
& =-\frac{1}{2} \varepsilon^{k r s}\left(\omega_{X}\right)_{i k}\left(\omega_{Y}\right)_{r s}=\left(\omega_{X}\right)_{i k} Y^{k} .
\end{aligned}
$$

Application of $\pi_{1}$ to the adjoint map $\operatorname{Ad}_{\exp \omega}: \mathfrak{s o}(3) \rightarrow \mathfrak{s o}(3)$ gives

$$
\begin{aligned}
\pi_{1} \circ \exp \theta \operatorname{ad}_{\omega_{\bar{X}}}\left(\omega_{Y}\right) & =\pi_{1} \circ\left(I+\sin \theta \operatorname{ad}_{\omega_{\bar{X}}}+(1-\cos \theta) \operatorname{ad}_{\omega_{\bar{X}}}^{2}\right) \omega_{Y} \\
& =\left(I+\sin \theta \omega_{\bar{X}}+(1-\cos \theta) \omega_{\bar{X}}^{2}\right) Y=R(\theta, \bar{X}) Y
\end{aligned}
$$

with $R(\theta, \bar{X})$ given by $(7)$. A similar results is obtained for the adjoint operator $\operatorname{Ad}_{\exp u_{\bar{X}}}$ on $\mathfrak{s u}(2)$ using $\pi_{2}$

$$
\begin{aligned}
\pi_{2} \circ \exp \theta \operatorname{ad}_{u_{\bar{X}}}\left(u_{Y}\right) & =\pi_{2} \circ\left(I+\sin \theta \operatorname{ad}_{u_{\bar{X}}}+(1-\cos \theta) \operatorname{ad}_{u_{\bar{X}}}^{2}\right) u_{Y} \\
& =R(\theta, \bar{X}) Y .
\end{aligned}
$$


Rotations of three-dimensional vectors are thus images of the group actions of $\mathrm{SO}(3)$ and $\mathrm{SU}(2)$ on their respective algebras $\mathfrak{s o}(3)$ and $\mathfrak{s u}(2)$. Hence from the LIE algebraic point of view a rotation of $Y \in \mathbb{R}^{3}$ about an axis $X \in \mathbb{R}^{3}$ is described as $\mathrm{SO}(3)$ and as $\mathrm{SU}(2)$ action with $R=\exp \omega_{X}$ and $U=\exp u_{X}$ given respectively by

$$
\begin{array}{ll}
\omega_{Y^{\prime}}=\omega_{R Y}=\operatorname{Ad}_{R}\left(\omega_{Y}\right), & \omega_{Y} \in \mathfrak{s o}(3) \\
u_{Y^{\prime}}=u_{R Y}=\operatorname{Ad}_{U}\left(u_{Y}\right), & u_{Y} \in \mathfrak{s u}(2) .
\end{array}
$$

The vector form is then obtained via $\pi_{1}$ or $\pi_{2}$ where the product representation $R Y$ is a special feature of $\mathrm{SO}(3)$ due to the fact that $\pi_{1} \circ \operatorname{Ad}_{R}\left(\omega_{Y}\right)=R Y$. It is worth noting that rotations can be completely described using the associated LIE algebra elements $\omega_{X}, \omega_{Y}$ or $u_{X}, u_{Y}$.

The algebra isomorphisms $\pi_{3}: \mathfrak{s u}(2) \rightarrow \mathfrak{s o}(3)$ locally determines a group homomorphism of $\mathrm{SU}(2)$ and $\mathrm{SO}(3)$. As already seen $\pi_{1} \circ \operatorname{Ad}_{\exp \theta \omega_{\bar{X}}} \omega_{Y}=\exp \left(\theta \omega_{\bar{X}}\right) Y$ $=R(\theta, \bar{X})$ and $\operatorname{Ad}_{\exp \theta u_{\bar{X}}} v_{Y}=\exp \left(\theta u_{\bar{X}}\right) v_{Y} \exp \left(-\theta u_{\bar{X}}\right)$ describe the same rotation. Because $\operatorname{det}\left(\operatorname{Ad}_{\exp \theta u_{\bar{X}}} u_{Y}\right)=\operatorname{det} u_{Y}=\|Y\|^{2}$ this transformation is orthogonal and thus to each $U=\mathrm{e}^{\theta u_{X}} \in \mathrm{SU}(2)$ can be uniquely assigned a $R \in \mathrm{SO}(3)$ via the homomorphism $\Pi_{3}: \mathrm{SU}(2) \rightarrow \mathrm{SO}(3)$

$$
\Pi_{3}: \mathrm{e}^{\theta u_{X}} \mapsto \mathrm{e}^{\theta \omega_{X}}
$$

The map $\Pi_{3}$ is surjective but not injective since, as can be deduced from (41) with $\pi_{2}$ any two elements $\pm U \in \mathrm{SU}(2)$ describe the same rotation. However, $\Pi_{3}$ is a group isomorphism of $\mathrm{SO}(3)$ and the projective special unitary group $P \mathrm{SU}(2):=$ $\mathrm{SU}(2) /\{I,-I\} \cong \mathrm{SO}(3)$. Especially the generators $L_{i}$ and $E_{i}$ of $\mathfrak{s o}(3)$ and $\mathfrak{s u}(2)$ respectively generate one parametric (and hence ABELian) subgroups and the isomorphism $\Pi_{3}: P S U(2) \rightarrow \mathrm{SO}(3)$ yields the following relations between elementary rotations

$$
\begin{aligned}
& U_{1}(\theta):=\exp \theta E_{1} \\
& R_{1}(\theta):=\exp \theta L_{1} \\
& =\left(\begin{array}{cc}
\cos \frac{\theta}{2} & \mathrm{i} \sin \frac{\theta}{2} \\
\mathrm{i} \sin \frac{\theta}{2} & \cos \frac{\theta}{2}
\end{array}\right) \quad \stackrel{\Pi_{3}}{\longrightarrow} \quad=\left(\begin{array}{ccc}
1 & 0 & 0 \\
0 & \cos \theta & -\sin \theta \\
0 & \sin \theta & \cos \theta
\end{array}\right)
\end{aligned}
$$




$$
\begin{aligned}
& U_{2}(\theta):=\exp \theta E_{2} \\
& R_{2}(\theta):=\exp \theta L_{2} \\
& =\left(\begin{array}{cc}
\cos \frac{\theta}{2} & -\sin \frac{\theta}{2} \\
\sin \frac{\theta}{2} & \cos \frac{\theta}{2}
\end{array}\right) \quad \stackrel{\Pi_{3}}{\longrightarrow} \\
& =\left(\begin{array}{ccc}
\cos \theta & 0 & \sin \theta \\
0 & 1 & 0 \\
-\sin \theta & & \cos \theta
\end{array}\right) \\
& U_{3}(\theta):=\exp \theta E_{3} \\
& R_{3}(\theta):=\exp \theta L_{3} \\
& =\left(\begin{array}{cc}
\mathrm{e}^{\mathrm{i} \frac{\theta}{2}} & 0 \\
0 & \mathrm{e}^{-\mathrm{i} \frac{\theta}{2}}
\end{array}\right) \\
& \stackrel{\Pi_{3}}{\longrightarrow} \\
& =\left(\begin{array}{ccc}
\cos \theta & -\sin \theta & 0 \\
\sin \theta & \cos \theta & 0 \\
0 & 0 & 1
\end{array}\right) .
\end{aligned}
$$

While the algebra isomorphism $\pi_{3}$ is global, $\Pi_{3}$ is only a local group homomorphism of $\mathrm{SU}(2)$ and $\mathrm{SO}(3)$ caused by the non-simply connectedness of $\mathrm{SO}(3)$ and $\mathrm{SU}(2)$ being its double covering [1]. It should be noticed that the basis (3) yields inverse rotation matrices, i.e., describing counterclockwise rotations about $X$ as it is obvious from $(3)$.

Having established an isomorphism of $\mathrm{SO}(3)$ and $\mathrm{SU}(2)$ enables to describe rotations either using a chart on $\mathrm{SO}(3)$ or on $\mathrm{SU}(2)$ with $\mathrm{C} 1 \mathrm{~K}$ or $\mathrm{C} 2 \mathrm{~K}$ respectively. With Proposition 3 the EP are given in terms of a unit rotation axis $\bar{X} \in \mathbb{R}^{3}$ and angle $\theta$ or in terms of $X \in \mathbb{R}^{3}$ by

$$
\begin{array}{lll}
p^{0}=\cos \frac{\theta}{2}, & p^{i}=\bar{X}^{i} \sin \frac{\theta}{2}, & i=1,2,3, \quad-\pi<\theta \leq \pi \\
p^{0}=\cos \frac{\|X\|}{2}, & p^{i}=\frac{X^{i}}{\|X\|} \sin \frac{\|X\|}{2}, & i=1,2,3, \quad-\pi<\|X\| \leq \pi .
\end{array}
$$

The isomorphism $\pi_{3}=\pi_{1}^{-1} \circ \pi_{2}$ applied to (41) and (42) yields $R(p) Y=$ $\pi_{2} \circ \operatorname{Ad}_{\exp u_{X}} v_{Y}=\pi_{1} \circ \operatorname{Ad}_{\exp \omega_{X}} \eta_{Y}=\exp \left(\omega_{X}\right) Y=R(X) Y$ with

$$
\begin{aligned}
R(p) Y= & \pi_{2} \circ\left(\begin{array}{cc}
p^{0}+\mathrm{i} p^{3} & p^{2}+\mathrm{i} p^{1} \\
-p^{2}+\mathrm{i} p^{1} & p^{0}-\mathrm{i} p^{3}
\end{array}\right) \cdot\left(\begin{array}{cc}
\mathrm{i} Y^{1} & Y^{2}+\mathrm{i} Y^{3} \\
-Y^{2}+\mathrm{i} Y^{3} & -\mathrm{i} Y^{1}
\end{array}\right) \\
& \cdot\left(\begin{array}{cc}
p^{0}-\mathrm{i} p^{3} & -p^{2}-\mathrm{i} p^{1} \\
p^{2}-\mathrm{i} p^{1} & p^{0}+\mathrm{i} p^{3}
\end{array}\right)
\end{aligned}
$$

such that with the unitarity condition $\sum_{\nu=0}^{3}\left(p^{\nu}\right)^{2}=1 \operatorname{due}$ to $\operatorname{det} U=1$

$$
\begin{aligned}
R(p) & =\left(\begin{array}{ccc}
\frac{1}{2}\left(a^{2}-b^{2}+\bar{a}^{2}-\bar{b}^{2}\right) & -\frac{\mathrm{i}}{2}\left(a^{2}+b^{2}-\bar{a}^{2}-\bar{b}^{2}\right) & -a b-\bar{a} \bar{b} \\
\frac{\mathrm{i}}{2}\left(a^{2}-b^{2}-\bar{a}^{2}+\bar{b}^{2}\right) & \frac{1}{2}\left(a^{2}+b^{2}+\bar{a}^{2}+\bar{b}^{2}\right) & \mathrm{i}(\bar{a} \bar{b}-a b) \\
a \bar{b}+\bar{a} b & \mathrm{i}(\bar{a} b-a \bar{b}) & a \bar{a}-b \bar{b}
\end{array}\right) \\
& =\left(\begin{array}{ccc}
1-2\left[\left(p^{2}\right)^{2}-\left(p^{3}\right)^{2}\right] & 2\left(p^{1} p^{2}-p^{0} p^{3}\right) & 2\left(p^{1} p^{3}+p^{0} p^{2}\right) \\
2\left(p^{1} p^{2}+p^{0} p^{3}\right) & 1-2\left[\left(p^{1}\right)^{2}-\left(p^{3}\right)^{2}\right] & 2\left(p^{2} p^{3}-p^{0} p^{1}\right) \\
2\left(p^{1} p^{3}-p^{0} p^{2}\right) & 2\left(p^{2} p^{3}+p^{0} p^{1}\right) & 1-2\left[\left(p^{1}\right)^{2}-\left(p^{2}\right)^{2}\right]
\end{array}\right)
\end{aligned}
$$


is a representation of rotation matrices in terms of EP. The multiplication law of $\mathrm{EP}$ is naturally defined by that of $\mathrm{SU}(2)$ matrices which is exactly the multiplication law of quaternion [1] since EP are nothing but normalized quaternions. Let $W=U V$ be given as product of two $\mathrm{SU}(2)$ matrices described by their associated $\mathrm{EP} p_{U}^{\nu}$ and $p_{V}^{\nu}$ then EP of $W$ are given by

$$
\begin{aligned}
& p_{W}^{0}=p_{U}^{0} p_{V}^{0}-\sum_{i=1,2,3} p_{U}^{i} p_{V}^{i} \\
& p_{W}^{i}=p_{U}^{0} p_{V}^{i}+p_{V}^{0} p_{U}^{i}+\varepsilon^{i}{ }_{j k} p_{U}^{j} p_{V}^{k}, \quad i=1,2,3 .
\end{aligned}
$$

Using $\mathrm{C} 2 \mathrm{~K}$ to describe spatial rotations by successively applying elementary rotations is equivalent to a local representation of $\mathrm{SO}(3)$ or $\mathrm{SU}(2)$ as product of three one-dimensional subgroups. EULER and BRYANT angle descriptions are two commonly used examples. With $\mathrm{C} 2 \mathrm{~K} \mathrm{SO}(3)$ and $\mathrm{SU}(2)$ elements have the form

$$
\begin{array}{rlrl}
R & =R_{i}\left(\theta_{1}\right) R_{j}\left(\theta_{2}\right) R_{k}\left(\theta_{3}\right), & & R_{i}(\theta)=\exp \left(\theta L_{i}\right) \\
U=U_{i}\left(\theta_{1}\right) U_{j}\left(\theta_{2}\right) U_{k}\left(\theta_{3}\right), & & U_{i}(\theta)=\exp \left(\theta E_{i}\right), \quad i \neq j \neq k
\end{array}
$$

where a certain choice of $i, j, k$ yields a certain parametrization. An EULER angle parametrization of $\mathrm{SO}(3)$ and $\mathrm{SU}(2)$ with $\psi, \vartheta, \varphi$ describing successive rotations about the actual 3-1-3-axes yields (with $s_{x}:=\sin x, c_{x}:=\cos x$ )

$$
\begin{aligned}
R(\psi, \vartheta, \varphi) & =R_{3}(\psi) R_{1}(\vartheta) R_{3}(\varphi) \\
& =\left(\begin{array}{ccc}
c_{\varphi} c_{\psi}-c_{\vartheta} s_{\varphi} s_{\psi} & -c_{\psi} s_{\varphi}-c_{\varphi} c_{\vartheta} s_{\psi} & s_{\psi} s_{\vartheta} \\
c_{\psi} c_{\vartheta} s_{\varphi}+c_{\varphi} s_{\psi} & c_{\varphi} c_{\psi} c_{\vartheta}-s_{\varphi} s_{\psi} & -c_{\psi} s_{\vartheta} \\
s_{\varphi} s_{\vartheta} & c_{\varphi} s_{\vartheta} & c_{\vartheta}
\end{array}\right) \\
U(\psi, \vartheta, \varphi) & =U_{3}(\psi) U_{1}(\vartheta) U_{3}(\varphi) \\
& =\left(\begin{array}{cc}
\mathrm{e}^{\frac{i}{2} \phi+\frac{i}{2} \psi} \cos \frac{\vartheta}{2} & \mathrm{ie}^{\frac{-i}{2} \phi+\frac{i}{2} \psi} \\
\mathrm{i}^{\frac{i}{2} \phi-\frac{i}{2} \psi} \sin \frac{\vartheta}{2} & \mathrm{e}^{\frac{-i}{2} \phi-\frac{i}{2} \psi} \cos \frac{\vartheta}{2}
\end{array}\right) .
\end{aligned}
$$

Thus the CAYLEY-KLEIN parameter and EP for an EULER angle parametrization are respectively

$$
\begin{aligned}
a & =\mathrm{e}^{\frac{\mathrm{i}}{2} \phi+\frac{\mathrm{i}}{2} \psi} \cos \frac{\vartheta}{2}, & b & =\mathrm{i}^{\frac{-\mathrm{i}}{2} \phi+\frac{\mathrm{i}}{2} \psi} \\
p^{0} & =\cos \frac{\varphi+\psi}{2} \cos \frac{\vartheta}{2}, & p^{1} & =\cos \frac{\varphi-\psi}{2} \sin \frac{\vartheta}{2} \\
p^{2} & =\sin \frac{\psi-\varphi}{2} \sin \frac{\vartheta}{2}, & p^{3} & =\sin \frac{\varphi+\psi}{2} \cos \frac{\vartheta}{2}
\end{aligned}
$$


and it is clear that the EP are periodic in $\theta$ with $4 \pi$ due the double covering of $\mathrm{SO}(3)$ by $\mathrm{SU}(2)$, i.e., there exist two $U \in \mathrm{SU}(2)$ and thus two sets of $p^{\nu}$ such that $\Pi_{3}(U)=R \in \mathrm{SO}(3)$. More precisely these are $\pm U$ and $\pm p^{\nu}$, which immediately follows from (45). Although EP arise from $\mathrm{C} 1 \mathrm{~K}$ they are not canonical coordinates, neither of the first nor of the second kind. Moreover, EP result from $\mathrm{C} 1 \mathrm{~K}$, axis and angle, via (44) and from $\mathrm{C} 2 \mathrm{~K}$ via (50) e.g.

\section{Vector Parametrization Based on CAYLEY transformations}

Thus far the rotation group $\mathrm{SO}(3)$ can be globally parameterized with canonical coordinates of the first kind or locally using canonical coordinates of the second kind. Alternatively the global parametrization with $\mathrm{C} 1 \mathrm{~K}$ can be substituted by the redundant set of EP. The later has the advantage that it yields a global parametrization which only contains rational functions. However, this is owed to the use of four dependent parameter. Obviously CP or RP also describes rotation matrices with rational functions and rotation matrices can be parameterized with the three RP. Furthermore the CP approach enables to assign a real parameter to each elementary rotation which is not possible with EP. Unfortunately the fact that the CAYLEY transformation on $\mathrm{SO}(3)$ cannot describe arbitrary rotations (no reflexion) precludes its general use. A possible alternative is to relate the CAYLEY transformation on $\mathrm{SO}(3)$ and that on $\mathrm{SU}(2)$ to obtain a global parametrization of $\mathrm{SO}(3)$ which only contains rational functions, i.e., a parametrization with modified RP or CP. This allows the use of the minimal number of three parameters to globally parameterize rotations only with rational functions. The fact that $\mathrm{CP}$ parameterize one dimensional subgroups also gives rise to a local parametrization with $\mathrm{CP}$. The actual RP or $\mathrm{CP}$ can be related to the $\mathrm{C} 1 \mathrm{~K}$ or $\mathrm{C} 2 \mathrm{~K}$ respectively.

Consider the orthogonal matrix $R\left(\omega_{X}\right) \in \mathrm{SO}(3)$ describing rotations about the axis $X \in \mathbb{R}^{3}$ and let $\omega_{X}=\pi_{1}^{-1}(X) \in \mathfrak{s o}(3)$ be the associated skew symmetric matrix. $R$ can either be generated from $\omega_{X}$ by the exp map or from some $\xi_{c}=\pi_{1}^{-1}(c) \in \mathfrak{s o}(3)$ with associated RoDRIGUES vector $c$ using the cay map

$$
R\left(\omega_{X}\right)=\exp \omega_{X}=\operatorname{cay}\left(\xi_{c}\right)=R\left(\xi_{c}\right), \quad \omega_{X}, \xi_{c} \in \mathfrak{s o}(3)
$$

Due to the analyticity of exp there exist a locally unique $\omega$ for every $R$. It is thus necessary that $\operatorname{Ad}_{R} \omega_{X}=\omega_{X}$ and $\operatorname{Ad}_{R} \xi_{c}=\xi_{c}$ and that $\omega_{X}$ and $\xi_{c}$ are related by

$$
\xi_{c}=\lambda \omega_{X}, \quad \lambda \in \mathbb{R} .
$$


Comparing (11) and (7) together with (52) yields the condition for $\lambda$

$$
\frac{2 \lambda}{1+\lambda^{2}\left\|\omega_{X}\right\|^{2}}=\frac{\sin \left\|\omega_{X}\right\|}{\left\|\omega_{X}\right\|} \omega_{X} \wedge \frac{2 \lambda^{2}}{1+\lambda^{2}\left\|\omega_{X}\right\|^{2}}=\frac{1-\cos \left\|\omega_{X}\right\|}{\left\|\omega_{X}\right\|^{2}}
$$

which is fulfilled by

$$
\lambda:=\frac{1}{\left\|\omega_{X}\right\|^{2}} \tan \left\|\frac{\omega_{X}}{2}\right\| .
$$

It thus follows the relation of the exp and the cay map on $\mathrm{SO}(3)$ and that of $X$ and the RODRIGUES vector $c$

$$
\exp \omega_{X}=\operatorname{cay}\left(\frac{\tan \left\|\frac{\omega_{X}}{2}\right\|}{\left\|\omega_{X}\right\|^{2}} \omega_{X}\right), \quad Y=\frac{1}{\|X\|^{2}} \tan \left\|\frac{X}{2}\right\| .
$$

Assumed $\left\|\omega_{\bar{X}}\right\|=1$ such that $R(\theta)=\exp \left(\theta \omega_{\bar{X}}\right)$ then (55) relates the $\mathrm{CP} \gamma$ and the rotation angle $\theta$

$$
\gamma=\tan \frac{\theta}{2}, \quad-\pi<\theta \leq \pi
$$

where the range of $\theta$ is mapped to the entire real line and $-\infty<\gamma<\infty$. It also immediately follows that the exp map (7) on $\mathrm{SO}(3)$ can be expressed in the form

$$
\exp \left(\theta \omega_{\bar{X}}\right)=\operatorname{cay}\left(\tan \frac{\theta}{2} \omega_{\bar{X}}\right)=I+\frac{2}{1+\tan ^{2} \frac{\theta}{2}}\left(\omega_{\bar{X}}+\omega_{\bar{X}}^{2}\right)
$$

that indeed containing transcendental functions. The transformation (56) is periodic in $\theta$ with a period of $\pi$ and maps $\theta$, with $|\theta|<\pi$ onto the parameter $\gamma$, with a range $|\gamma|<\infty$ so that for $\theta=\pi$ the map cay $\left(\tan \frac{\theta}{2} \omega_{\bar{X}}\right)$ is not continuous. Hence the CAYLEY transformation may describe arbitrary rotations except a reflexion about $\bar{X}$. Comparing (11) and (45) yields the relation of $\mathrm{CP}$ and the corresponding EP

$$
p^{0}=\frac{1}{\sqrt{1+\gamma^{2}}}, \quad p^{i}=\frac{\gamma}{\sqrt{1+\gamma^{2}}} X^{i}, \quad i=1,2,3 .
$$

The case of $\theta= \pm \pi$ corresponds to the limit $\gamma \rightarrow \pm \infty$ that gives the expected EULER-Parameter for $\theta= \pm \pi \lim _{\gamma \rightarrow \infty} p^{0}=0$ and $\lim _{\gamma \rightarrow \infty} p^{i}=X^{i}, i=1,2,3$. Hence the well known cay map on $\mathrm{SO}(3)$ yields a three-parametric description of rotations which, however, cannot be used to describe arbitrary rotations.

An alternative vector description can be achieved based on the CAYLEY map (32) on $\mathfrak{s u}(2)$. Here again the relation to the exp map is of particular interest. Let $a \in \mathbb{R}^{3}$ the modified RodRIGUES vector of $U \in \mathrm{SU}(2)$. Presumed that cay $\left(v_{a}\right)$ 
and $\exp \left(u_{X}\right)$ with $u_{X}, v_{a} \in \mathfrak{s u}(2)$ generate the same matrix $U=\operatorname{cay}\left(2 v_{a}\right)=$ $\exp \left(u_{X}\right)$ it is necessary that $v_{a}=\lambda u_{X}, \lambda \in \mathbb{R}$. Comparing (24) and (32) gives two conditions on $\lambda$

$$
\frac{4-\lambda^{2}\left\|u_{X}\right\|^{2}}{4+\lambda^{2}\left\|u_{X}\right\|^{2}}=\cos \frac{1}{2}\left\|u_{X}\right\| \wedge \frac{8 \lambda}{4+\lambda^{2}\left\|u_{X}\right\|^{2}}=\frac{2}{\left\|u_{X}\right\|} \sin \frac{1}{2}\left\|u_{X}\right\|
$$

which can be combined as easy to solve quadratic equation $\lambda^{2}+\frac{4}{\left\|u_{X}\right\|} \lambda \cot \frac{1}{2}\left\|u_{X}\right\|$ $-\frac{4}{\left\|u_{X}\right\|^{2}}=0$. The two solutions are

$$
\lambda_{1}=\frac{2}{\left\|u_{X}\right\|} \tan \frac{1}{4}\left\|u_{X}\right\|, \quad \lambda_{2}=-\frac{2}{\left\|u_{X}\right\|} \cot \frac{1}{4}\left\|u_{X}\right\| .
$$

Here $\lambda_{1}$ is generated by $\theta \in(-\pi, \pi]$ and $\lambda_{2}$ by $\theta \in(\pi, 2 \pi]$ so that by definition of $\theta=\left\|u_{X}\right\|$ the solution can be restricted to $\lambda_{1}$. The transformation from the $\mathrm{C} 1 \mathrm{~K}$ $X$ to the modified RodRIGUES vector $a$ and from the $\mathrm{C} 2 \mathrm{~K} \theta$ to the modified CP $\alpha$ should be diffeomorphic in the range of definition. The respective mappings for the RP and $\mathrm{CP}$ are

$$
\begin{aligned}
X \quad \mapsto \quad a=\frac{\tan \frac{1}{4}\|X\|}{\|X\|} X \\
\theta \quad \mapsto \quad \alpha=\tan \frac{1}{4} \theta
\end{aligned}
$$

which are in contrast to $(56)$ bijective and continuously differentiable and $-\pi<$ $\theta \leq \pi$ is mapped to $-1<\alpha \leq 1$. The periodicity follows by taking $\|X\|=\theta$ modulo $2 \pi$ or $\alpha$ modulo 2 respectively. A rotation matrix parametrized with $\alpha$ in (60) is thus periodic in $\alpha$ with a period of 2 . Similarly to $\mathrm{SO}(3)$ the cay and exp map are related by

$$
\exp \left(\theta u_{\bar{X}}\right)=\operatorname{cay}\left(2 \tan \frac{\theta}{4} u_{\bar{X}}\right)
$$

The expression (60) can also be obtained via stereographic projection [24]. The modified RODRIGUES vector is a scaled version of the WIENER-MILENKOVIC vector [2], defined as $\frac{4}{\|X\|} \tan \frac{\|X\|}{4} X$, also called the conformal rotation vector.

Comparing (33) and (23) yields the relation of $a$ and $\alpha$ with the EP

$$
\begin{aligned}
p^{0} & =\frac{1-\|a\|^{2}}{1+\|a\|^{2}} & p^{i} & =\frac{2}{1+\|a\|^{2}} a^{i} \\
p^{0} & =\frac{1-\alpha^{2}}{1+\alpha^{2}} & p^{i} & =\frac{2 \alpha}{1+\alpha^{2}} \bar{X}^{i} .
\end{aligned}
$$


Due to this relation the composition of modified RP is governed by the quaternion composition rule (46). Incorporating (63) into (45) yields a rotation matrix in terms of the modified RP $a$ or $\mathrm{CP} \alpha$

$$
\begin{aligned}
R(a) & =\Pi_{3} \circ \text { cay }\left(2 u_{a}\right)=I+\frac{4}{\left(1+\|a\|^{2}\right)^{2}}\left[\left(1-\|a\|^{2}\right) \omega_{a}+2 \omega_{a}^{2}\right] \\
R(\alpha, \bar{X}) & =\Pi_{3} \circ \text { cay }\left(2 \alpha u_{\bar{X}}\right) \\
& =I+\frac{4}{\left(1+\alpha^{2}\right)^{2}}\left[\alpha\left(1-\alpha^{2}\right) \omega_{\bar{X}}+2 \alpha^{2} \omega_{\bar{X}}^{2}\right]
\end{aligned}
$$

with $\omega_{a}=\pi_{1}^{-1}(a)$ and $\omega_{\bar{X}}=\pi_{1}^{-1}(\bar{X})$. A product representation of SU(2) and via $\Pi_{3}$ of $\mathrm{SO}(3)$ in terms of modified $\mathrm{CP}$ gives elements of the form

$$
R\left(\alpha_{1}, \alpha_{2}, \alpha_{3}\right)=R_{i}\left(\alpha_{1}\right) R_{j}\left(\alpha_{2}\right) R_{k}\left(\alpha_{3}\right), \quad i \neq j \neq k
$$

where $R_{i}(\alpha):=\Pi_{3}$ ocay $\left(2 \alpha E_{i}\right)$. A decomposition corresponding to the EULER angle decomposition is $R\left(\alpha_{1}, \alpha_{2}, \alpha_{3}\right)=R_{3}\left(\alpha_{1}\right) R_{1}\left(\alpha_{2}\right) R_{3}\left(\alpha_{3}\right)$, where the parameters are $\alpha_{1}=\tan \left(\frac{\psi}{4}\right), \alpha_{2}=\tan \left(\frac{\vartheta}{4}\right)$, and $\alpha_{3}=\tan \left(\frac{\varphi}{4}\right)$, and

$$
\begin{aligned}
& R_{1}(\alpha)=\left(\begin{array}{ccc}
1 & 0 & 0 \\
0 & \frac{1-6 \alpha^{2}+\alpha^{4}}{\left(1+\alpha^{2}\right)^{2}} & \frac{4 \alpha\left(\alpha^{2}-1\right)}{\left(1+\alpha^{2}\right)^{2}} \\
0 & \frac{-4 \alpha\left(\alpha^{2}-1\right)}{\left(1+\alpha^{2}\right)^{2}} & \frac{1-6 \alpha^{2}+\alpha^{4}}{\left(1+\alpha^{2}\right)^{2}}
\end{array}\right) \\
& R_{3}(\alpha)=\left(\begin{array}{ccc}
\frac{1-6 \alpha^{2}+\alpha^{4}}{\left(1+\alpha^{2}\right)^{2}} & \frac{4 \alpha\left(\alpha^{2}-1\right)}{\left(1+\alpha^{2}\right)^{2}} & 0 \\
\frac{-4 \alpha\left(\alpha^{2}-1\right)}{\left(1+\alpha^{2}\right)^{2}} & \frac{1-6 \alpha^{2}+\alpha^{4}}{\left(1+\alpha^{2}\right)^{2}} & 0 \\
0 & 0 & 1
\end{array}\right) .
\end{aligned}
$$

Hence a global singularity free three-parametric description is achieved by the EULER-RODRIGUES formula (7) using $\mathrm{C} 1 \mathrm{~K} X$ or via the coordinate transformation (60) by (64) using the modified RODRIGUES parameter $a$. The later has the advantage that it only contains rational functions like the EULER parameter description (45), which, however, needs four dependent parameter. Due to the use of three independent RODRIGUES parameter $a^{i}$ it allows to describes one parametric subgroups of $\mathrm{SO}(3)$ with rational functions only, like (67). Thus a product representation of $\mathrm{SO}(3)$ can be achieved using modified CAYLEY parameter as coordinates which are related to the $\mathrm{C} 2 \mathrm{~K}$ via (61). This, of course, leads to the problem of singularities of any three-parametric description using coordinates of 
the second kind. The main advantage of using modified RP or CP is the absence of transcendental functions that may achieve a dramatically increased computation performance as well a higher numerical accuracy with respect to numerical quantization error cumulation. The correspondence of the various representations of $\mathrm{SO}(3)$ are depicted in the following diagram

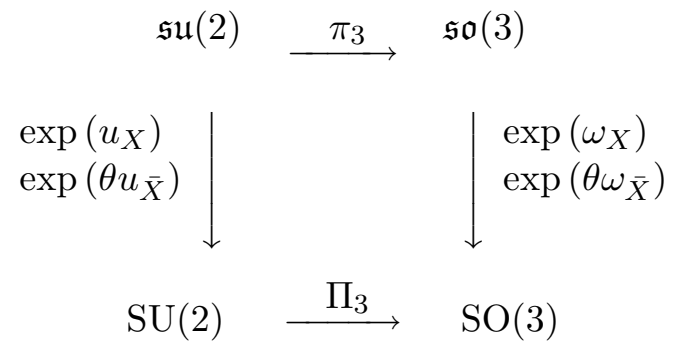

1. kind canonical coord. axis $X$, angle $\theta=\|X\|$ $\theta \in(-\pi, \pi]$

2. kind canonical coord. angle $\theta \in(-\pi, \pi]$

and those of $\mathrm{SU}(2)$ in the following diagram

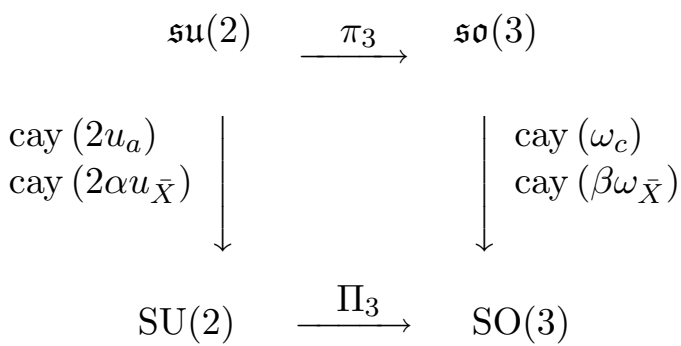

$$
\begin{array}{cc}
\begin{array}{c}
\text { modified RodRIGUES vector } \\
a=\frac{\tan \frac{1}{4}\|X\|}{\|X\|} X,\|a\| \in(-1,1]
\end{array} & \begin{array}{c}
\text { RodRIGUES parameter } \\
c=\frac{\tan \frac{1}{2}\|X\|}{\|X\|^{2}} X,\|c\| \in(-\infty, \infty)
\end{array} \\
\text { modified CAYLEY parameter } & \text { CAYLEY parameter } \\
\alpha=\tan \frac{1}{4} \theta, \alpha \in(-1,1] & \gamma=\tan \frac{1}{2} \theta, \gamma \in(-\infty, \infty) .
\end{array}
$$

Any specific parametrization determines a map from a three-dimensional parameter space to the group $\mathrm{SO}(3)$. This parameter space is moreover a differentiable manifold and hence any of the representations of rotation matrices defines a diffeomorphism from the parameter space to $\mathrm{SO}(3)$ as differentiable manifolds based on local charts. Coordinates in the respective chart are the minimal coordinates in the LAGRANGIAN motion equations of a rotating rigid body. For a freely rotating rigid body the parameter space is of dimension three but if the rigid body 
motion is restricted to a submanifold it is of lower dimension, e.g. in case of planar rotations. With $\mathrm{C} 1 \mathrm{~K} X$ and their derived RP $c$ and $a$ the parameter space is $\mathbb{R}^{3}$. The parameter space for a product representation with $\mathrm{C} 2 \mathrm{~K}$ is the usual three-dimensional torus. More precisely it is the three-dimensional torus modulo $2 \pi$ : $\mathbf{T}^{3}(2 \pi)$. The coordinate transformation $(61)$ projects $\mathbf{T}^{3}(2 \pi)$ onto the threedimensional real space $\mathbb{R}^{3}$ modulo 2 : $\mathbf{T}^{3}(2)$ as being the parameter space for a parametrization with modified $\mathrm{CP} \alpha$. On the other hand the three-dimensional torus $\mathbf{T}^{3}(2 \pi)$ is mapped to the entire $\mathbb{R}^{3}$ by (56) which is the parameter space for a parametrization with $\mathrm{CP} \gamma$. Finally the EP approach defines a diffeomorphism from the three-dimensional unit sphere $S^{3}$ to $\mathrm{SO}(3)$. Schematically the different approaches can be summarized as follows

$$
\begin{aligned}
\text { C1K: } \quad X \in \mathbb{R}^{3} & \rightarrow \exp \left(\omega_{X}\right) \\
\mathrm{RP}: \quad c \in \mathbb{R}^{3} & \rightarrow \operatorname{cay}\left(\omega_{c}\right) \\
\text { modified RP: } \quad a \in \mathbb{R}^{3} & \rightarrow R(a) \\
\text { C2K: } \quad\left(\theta_{1}, \theta_{2}, \theta_{3}\right) \in \mathbf{T}^{3}(2 \pi) & \rightarrow R_{i}\left(\theta_{1}\right) R_{j}\left(\theta_{2}\right) R_{k}\left(\theta_{3}\right) \\
\text { CP: } \quad\left(\gamma_{1}, \gamma_{2}, \gamma_{3}\right) \in \mathbb{R}^{3} & \rightarrow R_{i}\left(\gamma_{1}\right) R_{j}\left(\gamma_{2}\right) R_{k}\left(\gamma_{3}\right) \\
\text { modified RP: } \quad\left(\alpha_{1}, \alpha_{2}, \alpha_{3}\right) \in \mathbf{T}^{3}(2) & \rightarrow R_{i}\left(\alpha_{1}\right) R_{j}\left(\alpha_{2}\right) R_{k}\left(\alpha_{3}\right) \\
\text { EP: } \quad\left(p^{0}, p^{1}, p^{2}, p^{3}\right) \in S^{3} & \rightarrow R(p) .
\end{aligned}
$$

\section{Angular Velocity and Acceleration}

The description of rigid body dynamics using certain parametrization is based on relations that relate the angular velocity and acceleration vector to time derivatives of the respective parameter. Depending on the choosing coordinates the angular velocity tensor can be expressed in term of $\mathrm{C} 1 \mathrm{~K}$ or RP $c$ and $a$ as well as with $\mathrm{C} 2 \mathrm{~K}$ or $\gamma$ and $\alpha$. Explicit relations for EULER angles, RP and EP are reviewed in this section and relations for the modified RP and CP are derived. Consider the angular velocity tensor $\omega$ in body, or convective, representation and in spatial representation $\omega^{s}$ respectively [17]

$$
\omega=R^{-1} \dot{R}, \quad \omega^{s}=\dot{R} R^{-1}, \quad \omega, \omega^{s} \in \mathfrak{s o}(3) .
$$

This is equivalent to the conventional definition of $\omega$ since with the rotation matrix $E:=R^{T}$ and the definition $\omega=\dot{E}^{T} E=-\left(E^{T} \dot{E}\right)^{T}$ it is $\omega_{i j}=-\dot{E}_{i}{ }^{(i)} E_{j}{ }^{(i)}$ 
and thus $\omega_{i}=-\frac{1}{2} \varepsilon_{i j k} \omega_{j k}=\frac{1}{2} \varepsilon_{i j k} \dot{E}_{i}{ }^{(i)} E_{j}{ }^{(i)}$ are the components of the angular velocity vector [31]. The angular velocity vector is obtained via $\omega:=\pi_{1}(\omega)$.

Given the $\mathrm{C} 1 \mathrm{~K}$ representation (7) in terms of $X$ enables to express the angular velocity in terms of $\dot{X}$. Adopting the general result for the differential of the exponential map on a LIE -group $G$ with algebra $\mathfrak{g}$ [10], [25]

$$
\mathrm{d} \exp X=\left.\mathrm{d} L_{\exp X}\right|_{e} \circ \frac{1-\mathrm{e}^{-\mathrm{ad}_{X}}}{\operatorname{ad}_{X}}, \quad X \in \mathfrak{g}
$$

for the algebra $\mathfrak{s o}(3)$ yields with $R(X(t))=\exp X(t)$ and $L_{g} h=g h, g, h \in G$

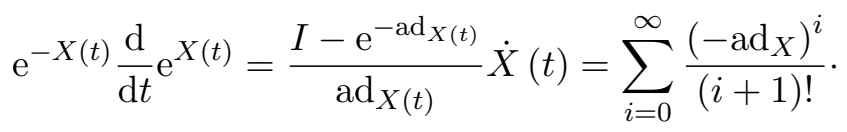

This together with (10) yields the angular velocity vector in terms of $\mathrm{C} 1 \mathrm{~K}$ on $\mathrm{SO}(3)$ and their time derivative

$$
\omega=\Omega_{X} \cdot \dot{X}, \text { with } \underset{X}{\Omega}(X)=I-\frac{1-\cos \|X\|}{\|X\|^{2}} \omega_{X}+\frac{\|X\|-\sin \|X\|}{\|X\|^{3}} \omega_{X}^{2} .
$$

If $R(c)=$ cay $\left(\omega_{c}\right)$ a direct evaluation of $\pi_{1}\left(R^{-1} \dot{R}\right)$ yields

$$
\omega=\underset{c}{\Omega} \cdot \dot{c}, \quad \text { with } \quad \underset{c}{\Omega}(c)=\frac{2}{1+\|c\|^{2}}\left(I-\omega_{c}\right) .
$$

A similar calculation relates time derivatives of modified RP $a$ to the angular velocity vector

$$
\omega=\underset{a}{\Omega} \cdot \dot{a}, \quad \text { with } \quad \underset{a}{\Omega}(a)=\frac{4}{1+\|a\|^{2}} I+\frac{8}{\left(1+\|a\|^{2}\right)^{2}}\left(\omega_{a}-\omega_{a}^{2}\right) .
$$

Obviously the image space of $\Omega_{X}$ and $\underset{a}{\Omega}$ is of constant dimension due to the general property $\operatorname{det} \underset{X}{\Omega}=1 \neq 0$, for all $X$ and $\operatorname{det} \underset{a}{\Omega}=\frac{64}{\left(1+\|a\|^{2}\right)^{3}} \neq 0,\|a\| \leq 1$ while $\operatorname{det} \underset{c}{\Omega}=\frac{8}{\left(1+\|c\|^{2}\right)^{2}} \stackrel{\|c\| \rightarrow \infty}{\longrightarrow} 0$ points toward a singularity of this map at $\|c\| \rightarrow \infty \triangleq\|X\| \rightarrow \pi$.

Let $R$ be given in the product representation (47) with C2K. Evaluating (67) with $\operatorname{Ad}_{R}(\eta)=R \omega R^{-1}$ yields

$$
\begin{aligned}
\omega & =\dot{\theta}_{i} \mathrm{e}^{-\theta_{k} L_{k}} \mathrm{e}^{-\theta_{j} L_{j}} L_{i} \mathrm{e}^{\theta_{j} L_{j}} \mathrm{e}^{\theta_{k} L_{k}}+\dot{\theta}_{j} \mathrm{e}^{-\theta_{k} L_{k}} L_{j} \mathrm{e}^{\theta_{k} L_{k}}+\dot{\theta}_{k} L_{k} \\
& =\dot{\theta}_{i} \mathrm{Ad}_{R_{j}\left(\theta_{j}\right) R_{k}\left(\theta_{k}\right)}^{-1}\left(L_{i}\right)+\dot{\theta}_{j} \mathrm{Ad}_{R_{k}\left(\theta_{k}\right)}^{-1}\left(L_{j}\right)+\dot{\theta}_{k} L_{k} .
\end{aligned}
$$


Specifically using EULER angles $(48)$ such that $R(\psi, \vartheta, \varphi)=R_{3}(\psi) R_{1}(\vartheta) R_{3}(\varphi)$ gives (with $s_{x}:=\sin x, c_{x}:=\cos x$ )

$$
\begin{aligned}
\omega & =\dot{\psi} \operatorname{Ad}_{R_{1}(\vartheta) R_{3}(\varphi)}^{-1}\left(L_{3}\right)+\dot{\vartheta} \operatorname{Ad}_{R_{3}(\varphi)}^{-1}\left(L_{1}\right)+\dot{\varphi} L_{3} \\
& =\left(\begin{array}{ccc}
0 & -\dot{\varphi}-\dot{\psi} c_{\vartheta} & -\dot{\vartheta} s_{\varphi}+\dot{\psi} c_{\varphi} s_{\vartheta} \\
\dot{\varphi}+\dot{\psi} c_{\vartheta} & 0 & -\dot{\vartheta} c_{\varphi}-\dot{\psi} s_{\varphi} s_{\vartheta} \\
\dot{\vartheta} s_{\varphi}-\dot{\psi} c_{\varphi} s_{\vartheta} & \dot{\vartheta} c_{\varphi}+\dot{\psi} s_{\varphi} s_{\vartheta} & 0
\end{array}\right)
\end{aligned}
$$

and hence the angular velocity vector $\omega:=\pi_{1}(\omega)$ is

$$
\omega=\Omega \cdot\left(\begin{array}{c}
\dot{\psi} \\
\dot{\vartheta} \\
\dot{\varphi}
\end{array}\right) \text {, with } \Omega(\psi, \vartheta, \varphi):=\left(\begin{array}{ccc}
\sin \varphi \sin \vartheta & \cos \varphi & 0 \\
\cos \varphi \sin \vartheta & -\sin \varphi & 0 \\
\cos \vartheta & 0 & 1
\end{array}\right) .
$$

The definition (67) of $\omega$ can be naturally extended to $\mathrm{SU}(2)$

$$
u=U^{-1} \dot{U}=U^{*} \dot{U}, \quad u \in \mathfrak{s u}(2)
$$

and with $\omega=\pi_{1}(\omega)=\pi_{2}(u)$ follows the relation of the time derivatives of EP and the angular velocity vector for the EP description (45) of $R$

$$
\omega=\underset{p}{\Omega} \cdot\left(\dot{p}^{0}, \dot{p}^{1}, \dot{p}^{2}, \dot{p}^{3}\right)^{T}
$$

with $\underset{p}{\Omega}\left(p^{0}, p^{1}, p^{2}, p^{3}\right):=2\left(\begin{array}{cccc}-p^{1} & p^{0} & p^{3} & p^{2} \\ -p^{2} & -p^{3} & p^{0} & p^{1} \\ -p^{3} & p^{2} & -p^{1} & p^{0}\end{array}\right)$.

Further if the rotation $R=\Pi_{3}(U)$ is described by the modified CP $\alpha$ the relation cay $\left(2 \alpha u_{\bar{X}}\right)=\exp \left(4 \arctan \alpha u_{\bar{X}}\right)$ yields

$$
\partial_{\alpha} \operatorname{cay}\left(2 \alpha u_{\bar{X}}\right)=\partial_{\alpha} \exp \left(4 \arctan \alpha u_{\bar{X}}\right)=\frac{4}{1+\alpha^{2}} \operatorname{cay}\left(2 \alpha u_{\bar{X}}\right)
$$

and $\dot{\theta}=\frac{4}{1+\alpha^{2}} \dot{\alpha}$. With $U=U_{i} U_{j} U_{k}$ expressed as product (47) yields similarly to (73)

$$
u=\dot{\theta}_{i} \operatorname{Ad}_{U_{j}\left(\theta_{j}\right) U_{k}\left(\theta_{k}\right)}^{-1}\left(E_{i}\right)+\dot{\theta}_{j} \operatorname{Ad}_{U_{k}\left(\theta_{k}\right)}^{-1}\left(E_{j}\right)+\dot{\theta}_{k} E_{k}
$$

and via $\pi_{2}$ the kinematic EULER equations (74) when using EULER angles. But if $U(\alpha, \bar{X})=$ cay $\left(2 \alpha u_{\bar{X}}\right)$ then

$$
u=\dot{\alpha}_{i} \frac{4}{1+\alpha_{i}^{2}} \operatorname{Ad}_{U_{j}\left(\alpha_{j}\right) U_{k}\left(\alpha_{k}\right)}^{-1}\left(E_{i}\right)+\dot{\alpha}_{j} \frac{4}{1+\alpha_{j}^{2}} \operatorname{Ad}_{U_{k}\left(\alpha_{k}\right)}^{-1}\left(E_{j}\right)+\dot{\alpha}_{k} \frac{4}{1+\alpha_{k}^{2}} E_{k} .
$$


Choosing the EULER composition $U=U_{3}\left(\alpha_{1}\right) U_{1}\left(\alpha_{2}\right) U_{3}\left(\alpha_{3}\right)$ and applying $\pi_{2}$ yields $\omega=\pi_{2}(u)$

$$
\begin{gathered}
\omega=\Omega_{\alpha} \cdot\left(\dot{\alpha}_{1}, \dot{\alpha}_{2}, \dot{\alpha}_{3}\right)^{T} \\
\underset{\alpha}{\Omega}\left(\alpha_{1}, \alpha_{2}, \alpha_{3}\right):=4\left(\begin{array}{ccc}
\frac{16 \alpha_{2}\left(\alpha_{2}^{2}-1\right) \alpha_{3}\left(\alpha_{3}^{2}-1\right)}{\left(4+\alpha_{1}^{2}\right)\left(4+\alpha_{2}\right)^{2}\left(4+\alpha_{3}^{2}\right)^{2}} & \frac{1-6 \alpha_{3}^{2}+\alpha_{3}{ }^{4}}{\left(1+\alpha_{2}{ }^{2}\right)\left(1+\alpha_{3}\right)^{2}} & 0 \\
-\frac{4 \alpha_{2}\left(\alpha_{2}^{2}-1\right)\left(1-6 \alpha_{3}^{2}+\alpha_{3}{ }^{4}\right)}{\left(1+\alpha_{1}^{2}\right)\left(1+\alpha_{2}\right)^{2}\left(1+\alpha_{3}^{2}\right)^{2}} & \frac{4 \alpha_{3}\left(\alpha_{3}{ }^{2}-1\right)}{\left(1+\alpha_{2}{ }^{2}\right)\left(1+\alpha_{3}\right)^{2}} & 0 \\
\frac{1-6 \alpha_{2}^{2}+\alpha_{2}{ }^{2}}{\left(1+\alpha_{1}^{2}\right)\left(1+\alpha_{2}\right)^{2}} & 0 & \frac{1}{1+\alpha_{3}{ }^{2}}
\end{array}\right) .
\end{gathered}
$$

It is worth noting that the factors in $R(\alpha)$ and $\underset{\alpha}{\Omega}$ are all powers of 2 . The singularity at $\alpha_{2}=0$ due to

$$
\operatorname{det} \underset{\alpha}{\Omega}=\frac{256 \alpha_{2}\left(\alpha_{2}^{2}-1\right)}{\left(1+\alpha_{1}^{2}\right)\left(1+\alpha_{2}^{2}\right)^{2}\left(1+\alpha_{3}^{2}\right)}
$$

corresponds to that for the EULER composition at $\vartheta=0 \operatorname{due}$ to $\operatorname{det} \Omega=-\sin \vartheta$. This singularity can be shifted using another chart for $T^{3}(2 \pi)$ or $T^{3}(2)$ respectively, e.g. using the BRYANT composition $R=R_{1}\left(\theta_{1}\right) R_{2}\left(\theta_{2}\right) R_{3}\left(\theta_{3}\right)$.

At any point the linear maps $\underset{X}{\Omega} \underset{a}{\Omega}, \underset{c}{\Omega}$ and $\underset{\alpha}{\Omega, \underset{\gamma}{\Omega}, \Omega_{\gamma}}$ and $\underset{p}{\Omega_{p}}$ are tangential maps from the tangent space of the respective parameter manifold to $\mathbb{R}^{3}$, e.g. $\Omega$ : $T \mathbf{T}^{3}(2 \pi) \rightarrow T \mathbb{R}^{3} \simeq \mathbb{R}^{3}$. These maps are the kinematic basic functions that appear in the LAGRANGIAN equations of motion for rigid multibody systems [16]. Lower dimensional rotations are described by restricting the number of parameters to one or two respectively.

The LAGRANGIAN motion equation of rigid bodies in minimal coordinates demand explicit relations of the angular acceleration in body representation and $\ddot{q}$. That is, $\dot{\omega}=\Omega \ddot{q}+\dot{\Omega} \dot{q}$, where $q$ is a vector of either one of the possible parameter sets, i.e., $q$ is the representing point on the parameter manifold. In the remaining part of this section only modified $\mathrm{CP} \alpha$ are considered in order clarify the problem of continuously differentiability of $\underset{a}{\Omega}$ and $\underset{\alpha}{\Omega}$. It holds with (47) and $U_{j}(\theta)=\exp \left(\theta E_{j}\right)$ that

$$
\begin{aligned}
\frac{\partial}{\partial \theta_{k}} \operatorname{Ad}_{U_{j} U_{k}}^{-1}\left(E_{i}\right) & =\left[\operatorname{Ad}_{U_{j} U_{k}}^{-1}\left(E_{i}\right), \operatorname{Ad}_{U_{k}}^{-1}\left(E_{j}\right)\right] \\
\frac{\partial}{\partial \theta_{k}} \operatorname{Ad}_{U_{k}}^{-1}\left(E_{j}\right) & =\left[\operatorname{Ad}_{U_{k}}^{-1}\left(E_{j}\right), E_{k}\right] .
\end{aligned}
$$


With $U_{j}(\alpha)=$ cay $\left(2 \alpha E_{j}\right)$ and $(77)$ follows that

$$
\begin{aligned}
\frac{\partial}{\partial \alpha_{j}} \operatorname{Ad}_{U_{j} U_{k}}^{-1}\left(E_{i}\right) & =\frac{4}{\left(1+\alpha_{i}^{2}\right)\left(1+\alpha_{j}^{2}\right)}\left[\operatorname{Ad}_{U_{j} U_{k}}^{-1}\left(E_{i}\right), \operatorname{Ad}_{U_{k}}^{-1}\left(E_{j}\right)\right] \\
\frac{\partial}{\partial \alpha_{k}} \operatorname{Ad}_{U_{k}}^{-1}\left(E_{j}\right) & =\frac{4}{\left(1+\alpha_{k}^{2}\right)\left(1+\alpha_{j}^{2}\right)}\left[\operatorname{Ad}_{U_{k}}^{-1}\left(E_{j}\right), E_{k}\right] .
\end{aligned}
$$

The time derivative of $\Omega_{\alpha}$ is thus given by

$$
\begin{aligned}
\underset{\alpha}{\dot{\Omega}=} & \pi_{2}\left[\frac{4 \dot{\alpha}_{j}}{\left(1+\alpha_{k}^{2}\right)\left(1+\alpha_{i}^{2}\right)^{2}}\left[\operatorname{Ad}_{U_{j} U_{k}}^{-1}\left(E_{i}\right), \operatorname{Ad}_{U_{k}}^{-1}\left(E_{j}\right)\right]\right. \\
& +\frac{4 \dot{\alpha}_{k}}{\left(1+\alpha_{k}^{2}\right)\left(1+\alpha_{j}^{2}\right)}\left[\operatorname{Ad}_{U_{k}}^{-1}\left(E_{j}\right), E_{k}\right] \\
& -\dot{\alpha}_{i} \frac{8 \alpha_{i}}{\left(1+\alpha_{i}^{2}\right)^{2}} \operatorname{Ad}_{U_{j} U_{k}}^{-1}\left(E_{i}\right)-\dot{\alpha}_{j} \frac{8 \alpha_{j}}{\left(1+\alpha_{j}^{2}\right)^{2}} \operatorname{Ad}_{U_{k}}^{-1}\left(E_{j}\right) \\
& \left.-\dot{\alpha}_{k} \frac{8 \alpha_{k}}{\left(1+\alpha_{k}^{2}\right)^{2}} E_{k}\right] .
\end{aligned}
$$

Similarly $\pi_{1}$ applied to (48) yields the well known relation of EULER angle and angular accelerations. This outlines the general approach for arbitrary compositions of elementary rotation. From the fact that the transformation (61) is of class $C^{1}$ follows that the tangential map $\Omega_{\alpha}: \dot{q} \rightarrow \omega$ is continuous but not continuously differentiable for $-1<\alpha \leq 1$. Especially the relation of $\ddot{q}$ and $\dot{\omega}$ is not continuous at $\alpha= \pm 1$ due to the last three terms in (85).

\section{Computational Aspects}

From the computational point of view vector like parametrization of rotations can potentially reduce the computational complexity and accuracy even on high performance computing facilities. These features stimulated the use of RP to describe the dynamics of rigid and flexible bodies. The RP parameterization naturally arises from the derivation of energy conserving integration schemes on the rotation group [15]. Also the algebraic nature of the rational parameterization with EP was addressed in the context of holonomic cut conditions for multibody systems [29] as well as for the efficient descriptions of the kinematics of robotic manipulators $[6,7]$. 


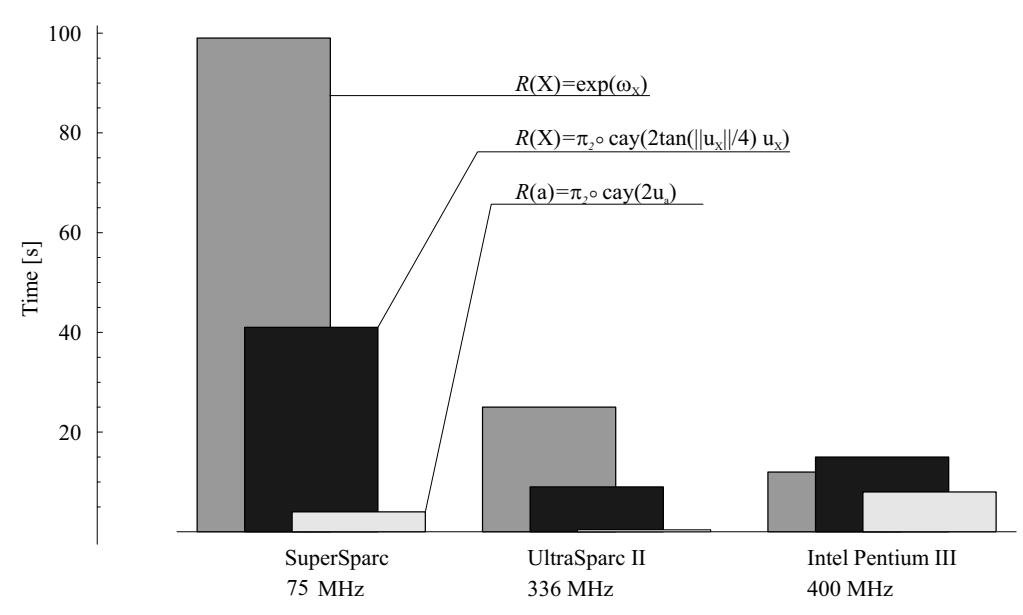

Figure 1. Evaluation time to obtain $R$ for $10^{6}$ different values of $\|X\| \in(-4 \pi, 4 \pi)$ and $\alpha \in(-4,4)$.

The absence of trigonometric functions which are a priory periodic demands to introduce a periodicity of $\alpha=\|a\|$ by definition. So $\alpha \in \mathbb{R}$ has to be mapped to $(-1,1]$ taking $\alpha$ modulo 2 , which can easily be carried out using simple shift operations in computer implementations. To exemplify the possible speed up we present Fig. 1 which shows the required time to evaluate a rotation matrix for $10^{6}$ different parameter values. Results are shown for $R(X)=\exp \left(\omega_{X}\right)$ in $(7)$ and $R(a)=\pi_{2} \circ$ cay $\left(u_{a}\right)$ in $(64)$ with $\|X\| \in(-2 \pi, 2 \pi)$ and $\alpha \in(-2,2)$ respectively. In situations where the use of $\theta=\|X\|$ is necessary then (64) can be used with (60) $a=\tan \frac{\|X\|}{4} \frac{X}{\|X\|}$ so that $R$ is parameterized as usual with $\theta$ but only one trigonometric functions is needed. The significant differences of the three considered computer architectures is the presence of a mathematical co-processor on the Pentium machine. Although the used computer hardware is not up-to-date the results apply to general architectures distinguished by the presence of a mathematical coprocessor, e.g. graphics processing unit (GPU) and other real-time hardware.

The globally singularity free three-parametric description of rotations with modified RP is owed to the lack of a continuously differentiable mapping from $\dot{a}$ to the angular velocity vector $\omega$. Because of $\dot{\theta}=\frac{4}{1+\alpha^{2}} \dot{\alpha}$ the transformation of time derivatives of $\alpha$ and $\theta$ is continuously but not continuously differentiable too, due to the discontinuity on the boundaries $\alpha= \pm 1$. This phenomenon indeed affects the integration process. To illustrate this effect the LAGRANGIAN motion equations for a disk rotating about a fixed axis with constant initial velocity 


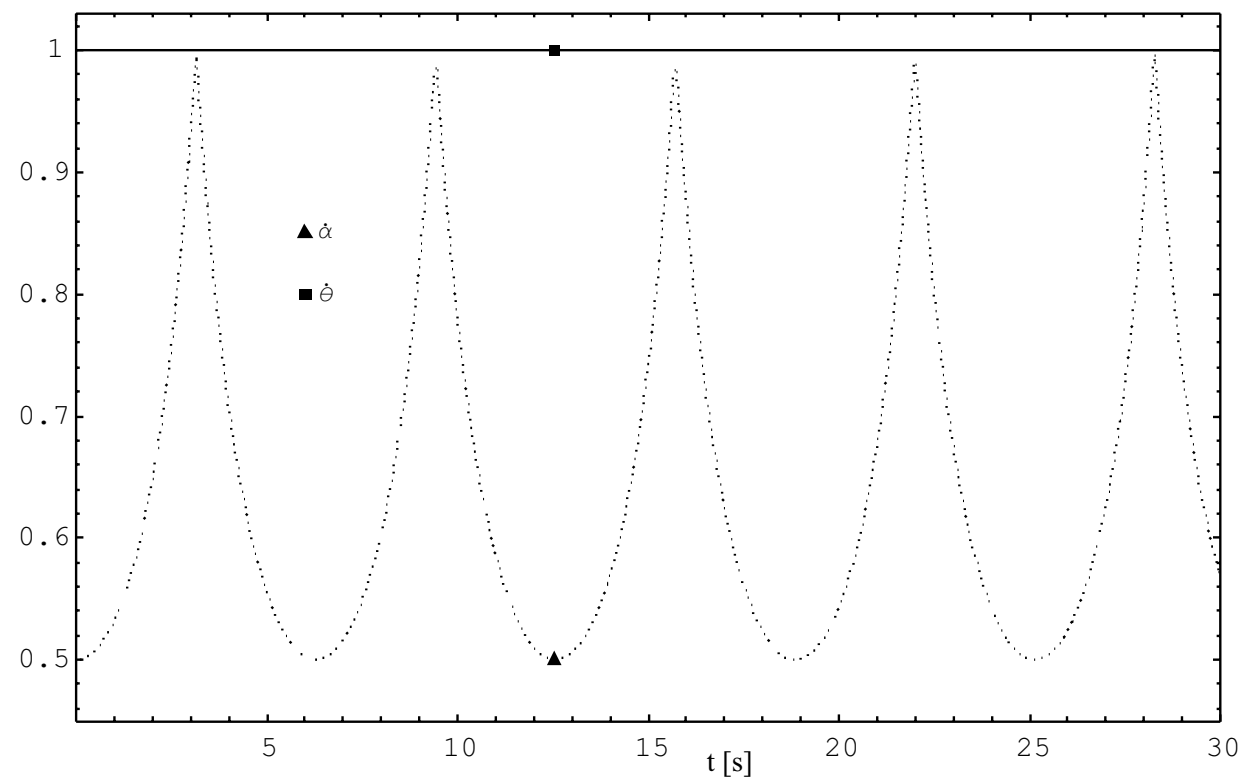

Figure 2. Time derivatives of $\theta$ and $\alpha$ for a disk rotating with constant angular velocity.

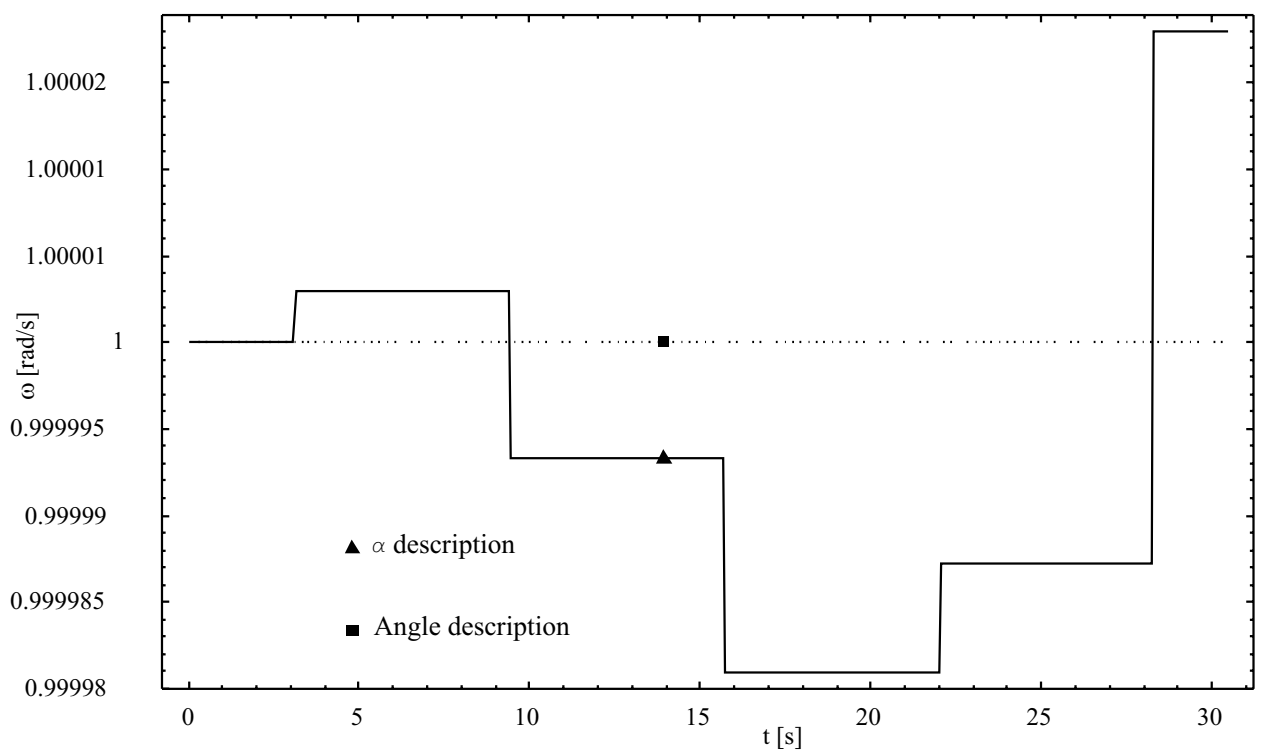

Figure 3. The drift of the angular velocity of the disc from remaining constant. 
and no external forces are integrated using a 4th order RUNGE-KUTTA algorithm with step size of $10^{-5} \mathrm{~s}$. The system with one degree of freedom was parameterized with angle $\theta$ and modified $\mathrm{CP} \alpha$ as respective generalized coordinates. Fig.2 shows the time evolution of the generalized velocities $\dot{\theta}$ and $\dot{\alpha}$ for the respective approach. The obvious discontinuity of $\dot{\alpha}$ explain the drift of the angular velocity from remaining constant as shown in Fig.3.

However, this phenomenon can be avoided for one and three-dimensional rotations since there exist a locally invertible relation of $\omega$ and $\dot{q} \in \mathbb{R}^{3}$ or $\dot{q} \in \mathbb{R}$. Using the state vector $(q, \omega)$ with $\dot{q}=\Omega^{-1} \omega$ the dynamics of rigid bodies is governed by the BOLZMANN-HAMEL equations (BHE) in non-holonomic velocity coordinates $\omega_{i}$ [16], [17]. For a freely rotating body these are precisely the dynamic EULER equations

$$
\Theta^{i j} \dot{\omega}_{j}+\varepsilon_{m n}^{i} \omega_{m} \Theta^{n j} \omega_{j}=0
$$

with $\omega(q)=\Omega(q) \dot{q}$ and $\Theta^{i j}$ being the inertia tensor. The main difference of the LAGRANGIAN motion equations and the BHE is that the LAGRANGE approach needs a transformation of class $C^{2}$ from the parameter manifold to the group $\mathrm{SO}(3)$ while the BHE equations only need a $C^{1}$ transformation from the configuration space to $\mathrm{SO}(3)$.

Since the RP $c$ and CP $\gamma$ are not even related to rotation matrices by a transformation of class $C^{0}$ they cannot be used in the LAGRANGE equations nor in the BHE. In case of the modified RP $a$ and $\mathrm{CP} \alpha$ the relation to $\omega$ is of class $C^{1}$ and for one and three-dimensional rotations a transformation from $\omega$ to $\dot{a}$ of class $C^{0}$ exists: $\dot{a}=\Omega_{a}^{-1} \omega$. This enables their use in the formulation of motion equations of rigid bodies with a degree of freedom one and three. Nevertheless in practical application, where rotations are restricted a priory, as it is the case for universal joints, the modified RP or CP are always applicable and the parameter range of $-1<\alpha \leq 1$ is more convenient than that of $\gamma$ which is $-\infty<\gamma<\infty$.

The CAYLEY transformation as being a first order approximation of the exponential map on $\mathrm{SO}(3)$ is employed for the update in integration schemes and for the approximation of finite rotations, e.g. in finite element methods. Also the cay-map on $\mathrm{SU}(2)$ is first order approximation of the exponential map and via the isomorphism (64) is a first order approximation of the exponential map on $\mathrm{SO}(3)$. The difference, however, is their parameter range and their behavior for small rotations. Comparing (55) and (54) and with the approximation of $\tan (\theta)$ the approximation error for the modified $\mathrm{CP} \alpha$ is smaller than that of the ordinary $\mathrm{CP} \gamma$ when approximating the exp map. 


\section{References}

[1] Altmann S., Rotations, Quaternions, and Double Groups, Oxford University Press, Oxford 1986.

[2] Bauchau O., Damilano G. and Theron N., Numerical Integration of Nonlinear Elastic Multi-Body Systems, Int. J. Num. Methods in Engineering 38 (1995) 272-2751.

[3] Cayley A., On the Motion of Rotation of a Solid, Cambridge and Dublin Mathematical Journal III (1843) 224-232.

[4] Cayley A., On Certain Results Relating to Quaternions, Phil. Mag. XXVL (1845) 141-145.

[5] Cayley A., On the Rotation of a Solid Body Round a Fixed Point, Cambridge and Dublin Mathematical Journal 1 (1846) 167-173 and 264-274.

[6] Chou J., Quaternion Kinematics and Dynamic Differential Equations, IEEE Trans. Robotics and Automation 8 (1992) 53-64.

[7] Chou J. and Kamel M., Finding the Position and Orientation of a Sensor on a Rrobot Manipulator using Quaternions, Int. Journal of Robotics Research 10 (1991) 240-254.

[8] Funda J., Taylor R. and Paul R., On Homogenous Transformations, Quaternions, and Computational Efficiency, IEEE Trans. Robotics and Automation 6 (1990) 382-388.

[9] Hamilton W., Some Applications of Quaternions to Questions Connected with the Rotation of a Solid Body, Royal Irish Academe Proceedings IV (1847-1850) 38-56.

[10] Helgason S., Differential Geometry, Lie Groups, and Symmetric Spaces, Academic Press, San Diego, 1978.

[11] Hall M., The Theory of Groups, MacMillan, New York, 1959.

[12] Ibrahimbegowic A. and Al Mikdad M., Finite Rotations in Dynamics of Beams and Implicit Time-Stepping Schemes, Int. J. Num. Methods in Engineering 41 (1998) 781-814.

[13] Klein F., Über die Bewegung des Kreisels, Nachrichten der Königlichen Geselschaft der Wissenschaften zu Göttingen vom 11. Januar 1896.

[14] Klein F., The Mathematical Theory of the Top, Lectures at Princeton University, October 1896. Both [13] and [14] In: Fricke R. and Vermel H. (Eds), Felix Klein, Gesammelte Mathematische Abhandlungen, Springer, Berlin 1922. 
[15] Lewis D. and Simo J., Conserving Algorithms for the Dynamics of Hamilton Systems on Lie Groups, J. Nonlinear Science 4 (1994) 253-299.

[16] Maißer P., A Differential Geometric Approach to the Multibody System Dynamics, Zeitschrift für Angew. Math. und Mech. 71 (1991) 116-119.

[17] Marsden J., Introduction to Mechanics and Symmetry, Springer, New York 1999.

[18] McCarthy J., Geometric Design of Linkages, Springer, New York, 2000.

[19] Mladenova C., Modelling and Control of Multibody Systems on a Configurational Space with a Lie Group Structure, J. Theor. Appl. Mech. 31 (2001) 3-25.

[20] Mladenova C., Group Theory in the Problems of Modeling and Control of Multi-Body Systems, JGSP 8 (2006) 17-121.

[21] Müller A. and Maißer P., A Lie-Group Formulation of Kinematics and Dynamics of Constrained MBS and Its Application to Analytical Mechanics, Multibody System Dynamics 9 (2003) 311-352.

[22] Murray R., Li Z. and Sastry S., A Mathematical Introduction to Robotic Manipulation, CRC Press, Boca-Raton, 1993.

[23] Normand J., A Lie Group: Rotations in Quantum Mechanics, North-Holland, Amsterdam, 1980.

[24] Tsiotras P., Junkins J. and Schaub H., Higher-Order Cayley Transformations with Applications to Attitude Representations, J. Guidance, Control and Dynamics 20 (1997) 528-534.

[25] Sattinger D. and Weaver O., Lie Groups and Algebras with Applications to Physics, Geometry and Mechanics, Springer, Berlin 1993.

[26] Siminovitch D., Rotations in NMR: Part I. Euler-Rodrigues Parameter and Quaternions, Concepts Magn. Reson. 9 (1997) 149-171.

[27] Siminovitch D., Rotations in NMR: Part II. Applications of the EulerRodrigues Parameter, Concepts Magn. Reson. 9 (1997) 211-225.

[28] Stuelpnagel J., On the Parametrization of the Three-Dimensional Rotation Group, SIAM Review 6 (1964) 422-430.

[29] Tasora A. and Righettini P., Application of Quaternion Algebra to the Efficient Computation of Jacobians for Holonomic-rheonomic Constraints, EUROMECH Colloquium 404, Advances in Computational Multibody Dynamics, 20-23 September 1999, pp.75-92.

[30] Vinberg E., Linear Representations of Groups, Birkhäuser, Basel, 1998. 
[31] Zihlin P., A New Approach to the Analysis of Free Rotations of Rigid Bodies, Zeitschrift für Angew. Math. und Mech. 4 (1996) 187-204.

\author{
Andreas Müller \\ University Duisburg-Essen \\ Chair of Mechanics and Robotics \\ 47057 Duisburg, GERMANY \\ E-mail address: andreas-mueller@uni-due.de
}

